\title{
Neonatal Cranial Ultrasound: Advanced Techniques and Image Interpretation
}

\author{
Erica L. Riedesel ${ }^{1}$ \\ ${ }^{1}$ Division of Pediatric Radiology, Department of Radiology, University \\ of Wisconsin School of Medicine and Public Health, Madison, \\ Wisconsin, United States \\ J Pediatr Neurol 2018;16:106-124.
}

\begin{abstract}
Address for correspondence Erica L. Riedesel, MD, Division of Pediatric Radiology, Department of Radiology, University of Wisconsin School of Medicine and Public Health, 600 Highland Avenue, Madison, WI 53792, United States

(e-mail: elriedesel@gmail.com; ERiedesel@uwhealth.org).
\end{abstract}

\begin{abstract}
Keywords

- cranial ultrasound

- Doppler

- B-flow imaging

- intraventricular hemorrhage

- germinal matrix hemorrhage

- hypoxic-ischemic injury

- ventriculomegaly

- meningitis

Neonatal cranial ultrasound has become an essential part of the routine care of neonates. Ultrasound is noninvasive, relatively inexpensive, and can be performed at the bedside. Addition of advanced ultrasound techniques such as use of high-frequency linear transducers, additional acoustic windows, and color and pulsed wave Doppler can significantly improve image quality and diagnostic accuracy of cranial ultrasound. This review will focus on these techniques and their application in the detection of central nervous system pathology in the high-risk preterm and term neonates.
\end{abstract}

\section{Introduction}

Neonatal cranial ultrasound has become an essential part of routine care in high-risk preterm and term neonates. Ultrasound is noninvasive, requires no sedation, and is relatively inexpensive, making it the ideal primary diagnostic screening tool for intracranial pathology. Most importantly, ultrasound can be performed at the bedside with minimal disturbance to the infant early in neonatal life and may be repeated as often as necessary for serial imaging of the brain. There are no contraindications for cranial ultrasound.

Neonatal cranial ultrasound may be performed at any time in the neonatal period. In preterm, $<32$ weeks' gestation, and extremely low birth weight (ELBW) infants $<1.5 \mathrm{~kg}$, an initial screening cranial ultrasound is suggested in the first 4 to 7 days of life with a repeat screening cranial ultrasound at 10 to 14 days. ${ }^{1}$ When used in this manner, cranial ultrasound has a near $100 \%$ sensitivity for the detection of severe intraventricular hemorrhage (IVH) and severe white matter injury. ${ }^{2}$ If initial screening ultrasound is normal, follow-up exam is suggested

received

March 1, 2017

accepted after revision

June 5, 2017

published online

August 14, 2017
Pediatric Neuroimaging; Guest Editor, Justin Brucker, MD

at corrected gestational age 36 to 40 weeks (term equivalent) to screen for evidence of more severe white matter injury. ${ }^{1}$ Findings on the term equivalent ultrasound in conjunction with magnetic resonance imaging (MRI) can be used to predict neurodevelopment outcomes.

In term infants, cranial ultrasound is suggested for evaluation of suspected intracranial hemorrhage, brain parenchymal abnormality, hypoxic-ischemic injury (HII), suspected ventriculomegaly, evaluation of congenital or acquired central nervous system (CNS) infection, and surveillance of previously documented abnormalities including prenatal abnormalities. ${ }^{1}$

\section{Basic Techniques in Neonatal Cranial Ultrasound}

Neonatal cranial ultrasound should be performed by an experienced sonographer familiar with normal ultrasound anatomy and the most frequently encountered anomalies of the neonatal brain. Details of basic technique and technical parameters have been established by the American College of

Copyright @ 2018 by Georg Thieme Verlag KG, Stuttgart · New York
DOI https://doi.org/ 10.1055/s-0037-1604488. ISSN $1304-2580$. 
Radiology, Society of Pediatric Radiology, Society of Radiologists in Ultrasound, and American Institute of Ultrasound in Medicine. ${ }^{1}$

Cranial ultrasound begins with grayscale imaging of the brain using a sector transducer via the anterior fontanel. The anterior fontanel, located in the calvarial vertex at the junction of the coronal and sagittal sutures, is the largest fontanel and provides an optimal sonographic window for global overview of the neonatal brain. The small footprint of the sector transducer is well matched to the size of the anterior fontanel. In addition, the relatively low ultrasound frequency $(8-5 \mathrm{MHz})$ of the sector transducer allows visualization of intracranial structures that are relatively deep to the anterior fontanel such as the brain stem and cerebellum.

In the coronal plane, images are obtained from anterior to posterior, beginning at the frontal lobes just anterior to the frontal horns of the lateral ventricles and extending to the occipital lobes posterior to the lateral ventricles. Imaging in the sagittal plane begins with a midline sagittal view that includes the corpus callosum, third and fourth ventricles, brain stem, and cerebellar vermis. Sequential images are then obtained through the right and left cerebral hemispheres, respectively, sweeping through the lateral ventricles, periventricular white matter, and peripheral cortex to the Sylvain fissures. ${ }^{1}$ Cine clips, in addition to representative still images, are helpful as complex or subtle findings may be better appreciated in the "real-time scanning" context. ${ }^{2,3}$

\section{Advanced Techniques in Neonatal Cranial Ultrasound}

\section{High-Frequency Linear Transducer}

Although the use of a low-frequency sector transducer allows a more global view of cerebral architecture, this comes at a loss of image resolution. In contrast, coronal and sagittal images through the anterior fontanel performed with a high-frequency linear transducer ( $9 \mathrm{MHz}$ or more) allows better resolution and more detailed visualization of structures in the near field and midline. ${ }^{2-5}$ This technique allows optimal evaluation of the deep gray structures of the basal ganglia, lateral ventricles, and periventricular white matter and allows more detailed assessment of gray-white matter differentiation in the cortex and subcortical white matter (-Fig. 1). This additional imaging significantly increases diagnostic capability of neonatal cranial ultrasound in both preterm and term infants.

By decreasing the depth of field and optimizing focal zone depth, high-frequency linear transducers may also be used for detailed evaluation of the superficial extra-axial space and the superior sagittal sinus just deep to the anterior fontanel (-Fig. 2)..$^{3-6}$

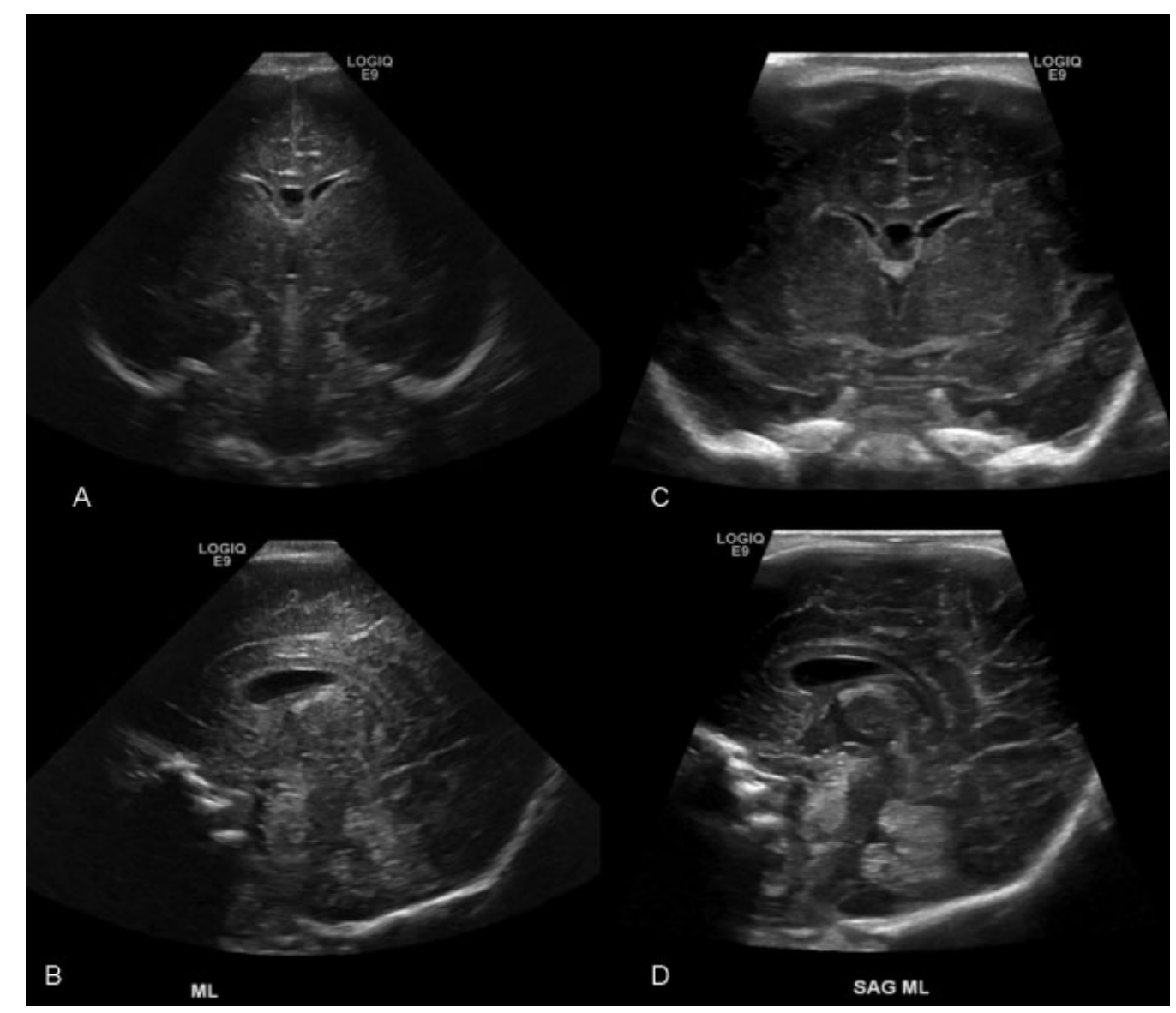

Fig. 1 Routine neonatal cranial ultrasound. (A, B) Coronal and sagittal planes with 7-MHz sector transducer and (C, D) coronal and sagittal planes with $9 \mathrm{MHz}$ linear transducer. Imaging with higher frequency transducer allows more detailed evaluation of brain anatomy and gray-white matter differentiation in the cortex and subcortical white matter. 


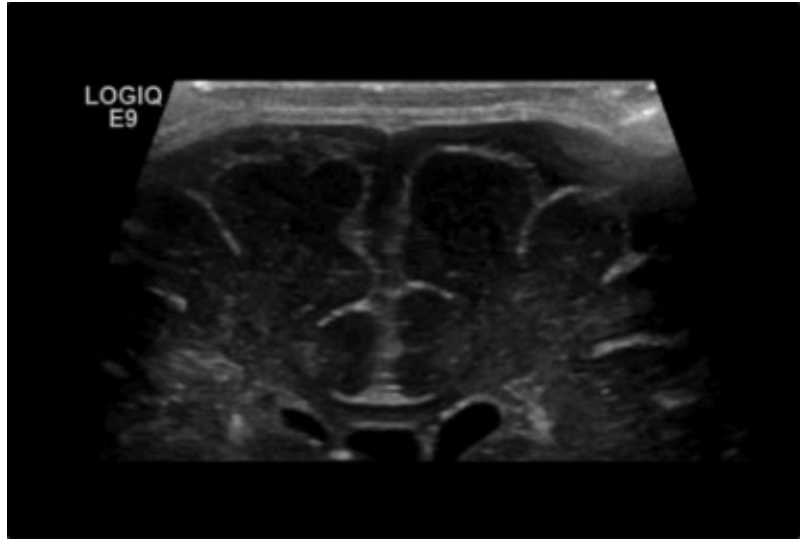

Fig. 2 Detailed evaluation of the superficial cortex, corpus callosum, and extra-axial space is possible with optimized depth of field and focal zone depth when imaging with high-frequency linear transducer.

\section{Doppler}

Color Doppler images obtained via the anterior fontanel or mastoid fontanel can be used to document normal intracranial vascular anatomy ( $\mathbf{- F i g . 3}$ ) or anatomic variants.

Imaging through the anterior fontanel in the sagittal plane provides an optimal view of the anterior cerebral artery (ACA) along the genu of the corpus callosum ( - Fig. 3A). Acquisition of spectral Doppler waveform at this site allows optimal assessment of peak systolic velocity (PSV), enddiastolic velocity (EDV), and resistive index (RI; defined as
PSV-EDV/PSV). ${ }^{3,7}$ The RI is influenced by many factors including flow velocity, cerebral blood flow volume, peripheral vascular resistance, and cerebral autoregulation. Thus, spectral Doppler evaluation of the intracranial arteries may provide important physiologic information in evaluation of the neonatal brain.

Relatively low intracerebral diastolic flow velocities in the premature circulation explain the relatively high ACA RI seen in the normal preterm brain of 0.7 to 0.8 (- Fig. 4A). ${ }^{2,7,8} \mathrm{As}$ the brain matures and the neonate undergoes physiologic transition from fetal to newborn intracerebral circulation, the normal ACA Doppler RI decreases to 0.7 to 0.75 ( - Fig. 4B). Over the first year of life, RI in the ACA will continue to decrease to a normal value of 0.6 in children aged 1 year and eventually reaches a normal value of 0.45 to 0.6 in children aged $>2$ years. ${ }^{2,7-9}$

The ACA RI will demonstrate changes in response to alterations in end-diastolic flow. For example, cerebral vascular dilatation in the setting of acute hypoxia or ischemia will result in increased end-diastolic flow generating a lower ACA RI ( - Fig. 5A). Conversely, in the setting of increased intracranial pressures (ICPs), end-diastolic flow will decrease generating a higher ACA RI ( - Fig. 5B). ${ }^{7,8}$

Special care in interpretation of ACA RI must be observed in neonates with cardiac disease. ${ }^{10}$ In these circumstances, especially in the setting of left-to-right shunt, end-diastolic flow in the intracerebral arteries will be variable. The most common example encountered in the neonatal intensive care

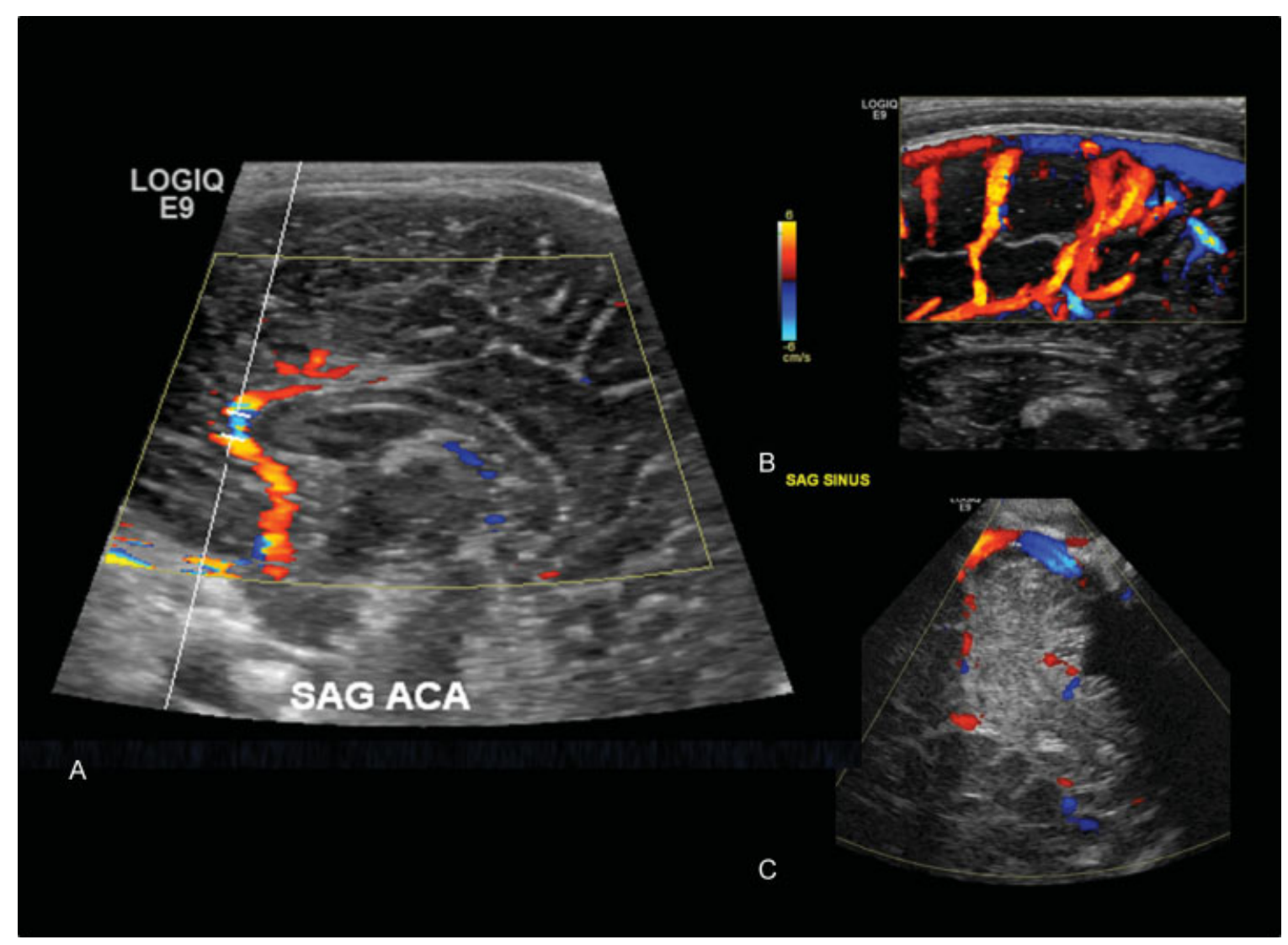

Fig. 3 Color Doppler may be used to document normal intracranial vascular anatomy including (A) Anterior cerebral artery and deep veins, (B) superior sagittal sinus imaged through the anterior fontanel, and (C) transverse sinus imaged through the mastoid fontanel. 


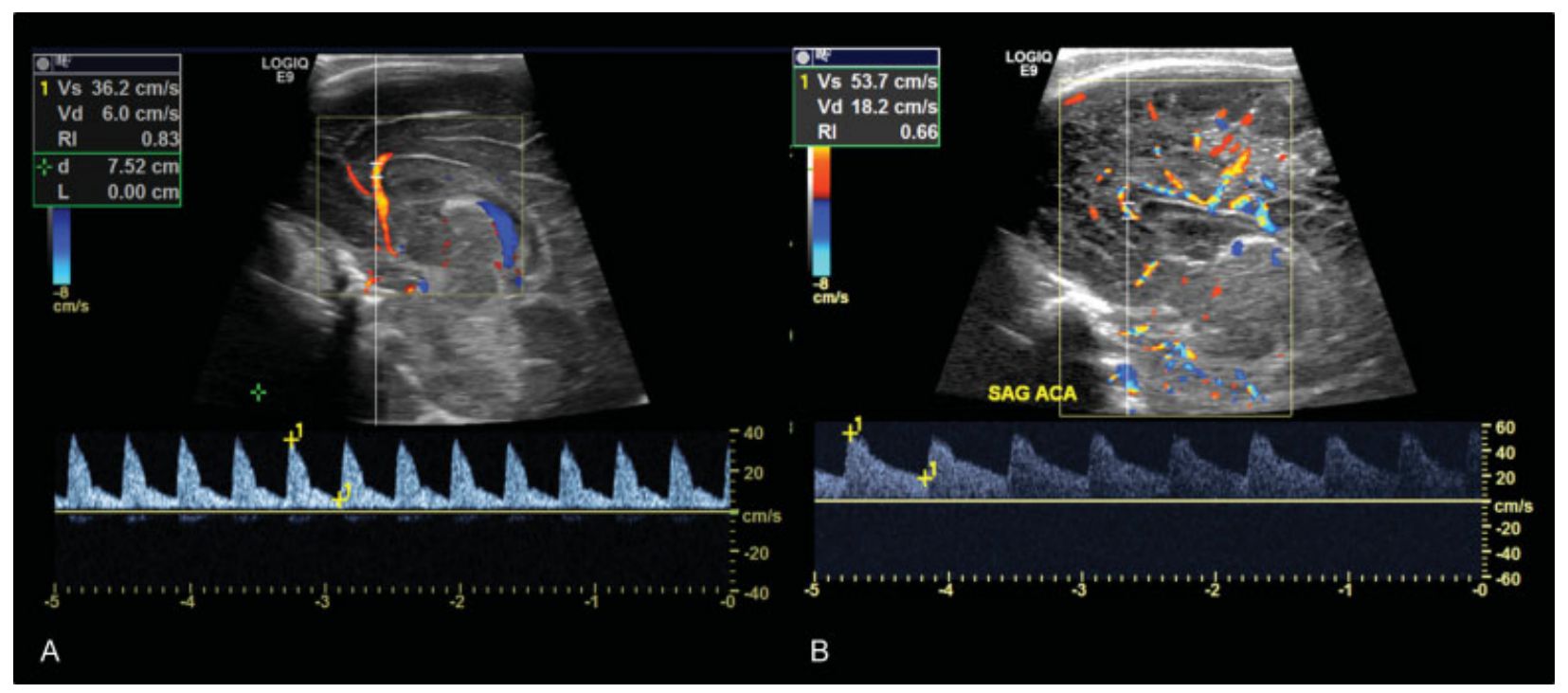

Fig. 4 Normal pulsed wave Doppler evaluation of ACA including RI in (A) preterm (34 weeks) and (B) term infant. ACA, anterior cerebral artery; $\mathrm{RI}$, resistive index.

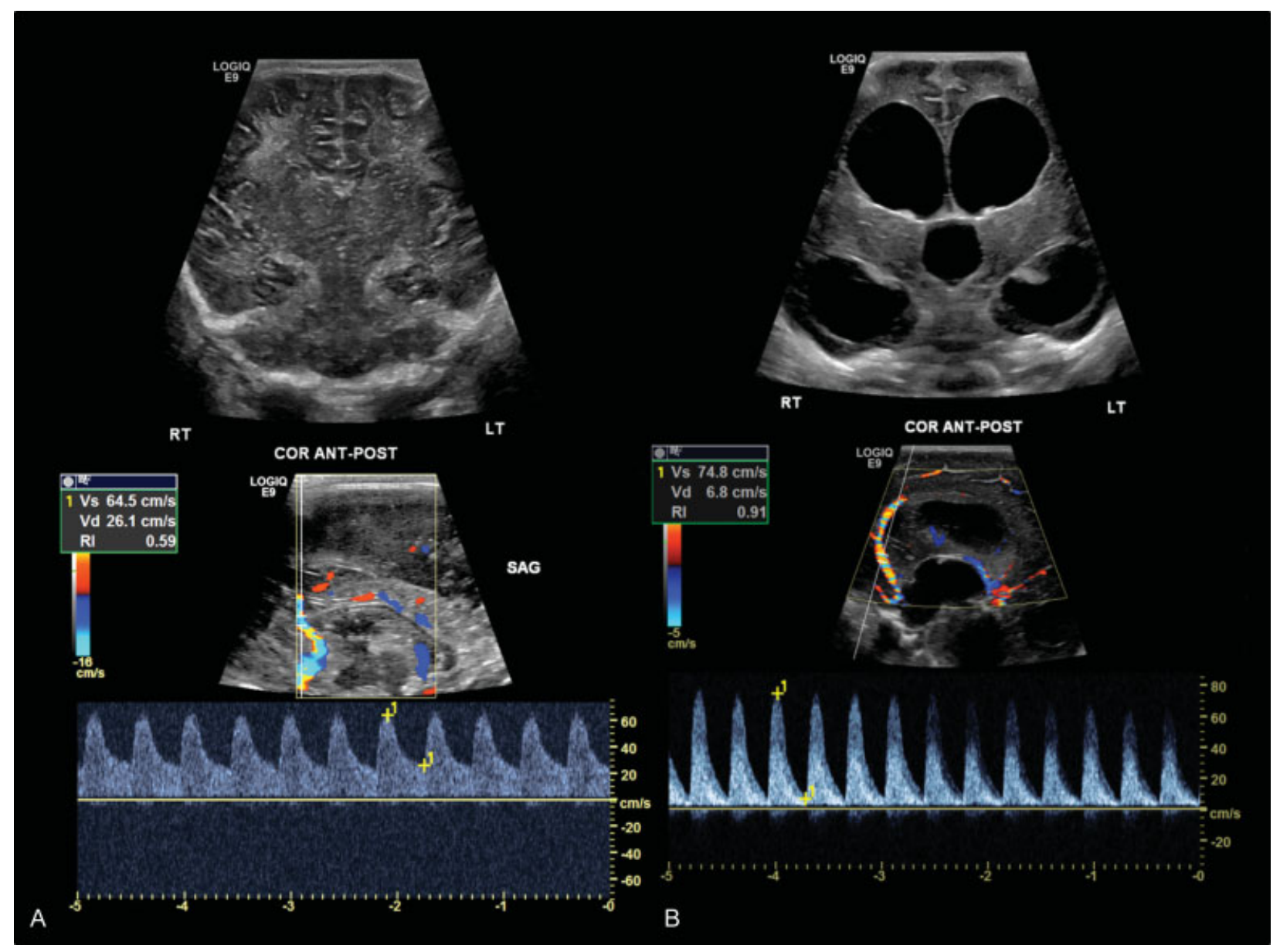

Fig. 5 Alterations of RI in the ACA reflect physiologic changes in intercerebral blood flow; (A) increased end-diastolic flow in the setting of acute hypoxic ischemic injury in term infant results in decreased RI; (B) decreased end-diastolic flow in the setting of increased ICP results in elevated RI. ACA, anterior cerebral artery; ICP, intracranial pressure; RI, resistive index. 


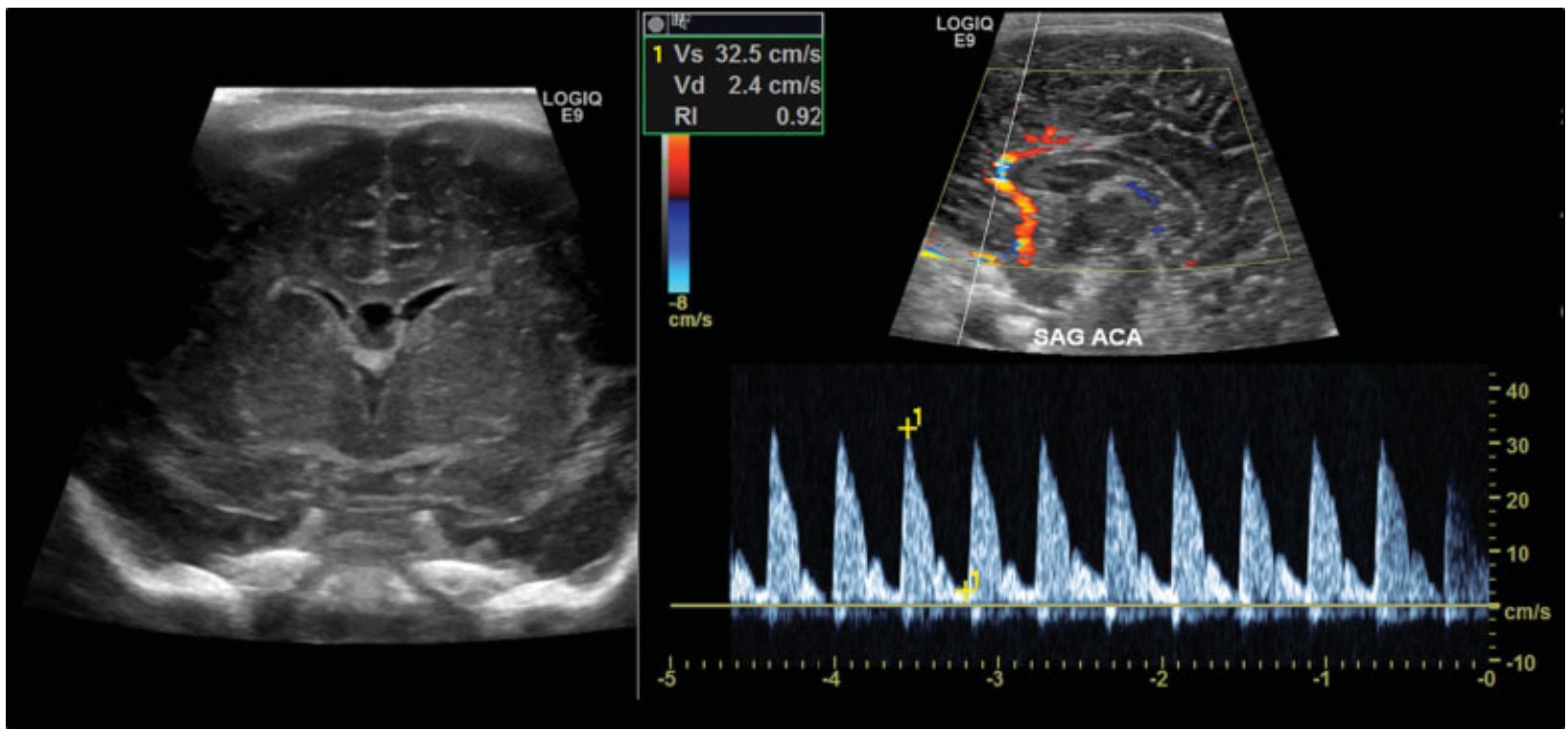

Fig. 6 ACA Doppler in term infant with PDA. Extracardiac left-to-right shunting results in decreased end-diastolic flow in the ACA. This should not be mistaken for elevated RI in the setting of increased intracranial pressure (note normal ventricular size and normal extra-axial spaces). ACA, anterior cerebral artery; PDA, patent ductus arteriosus; RI, resistive index.

unit is a patent ductus arteriosus (PDA). In a physiologically symptomatic PDA, end-diastolic flow in the ACA will be significantly decreased or even reversed (-Fig. 6), and this should not be mistaken for elevated ICP.

\section{B-Flow}

B-flow imaging is a non-Doppler technology that directly displays flowing intravascular echoes during real-time grayscale sonography. These images are especially impressive when viewed on real-time sonography or cine loops. B-flow imaging was first introduced on high-frequency linear transducers for superficial vascular imaging but has been quickly adopted into imaging of deeper vascular structures such as the portal vein.

The addition of B-flow imaging to neonatal cranial ultrasound can provide more detailed evaluation of intracranial vascular anatomy ( - Fig. 7). In addition, B-flow imaging can be used to detect regions of relative hyper- or hypovascularity in the setting of HII.

\section{Additional Acoustic Windows: Mastoid Fontanel}

The anterior fontanel provides an excellent acoustic window for supratentorial structures, although it is less optimal for

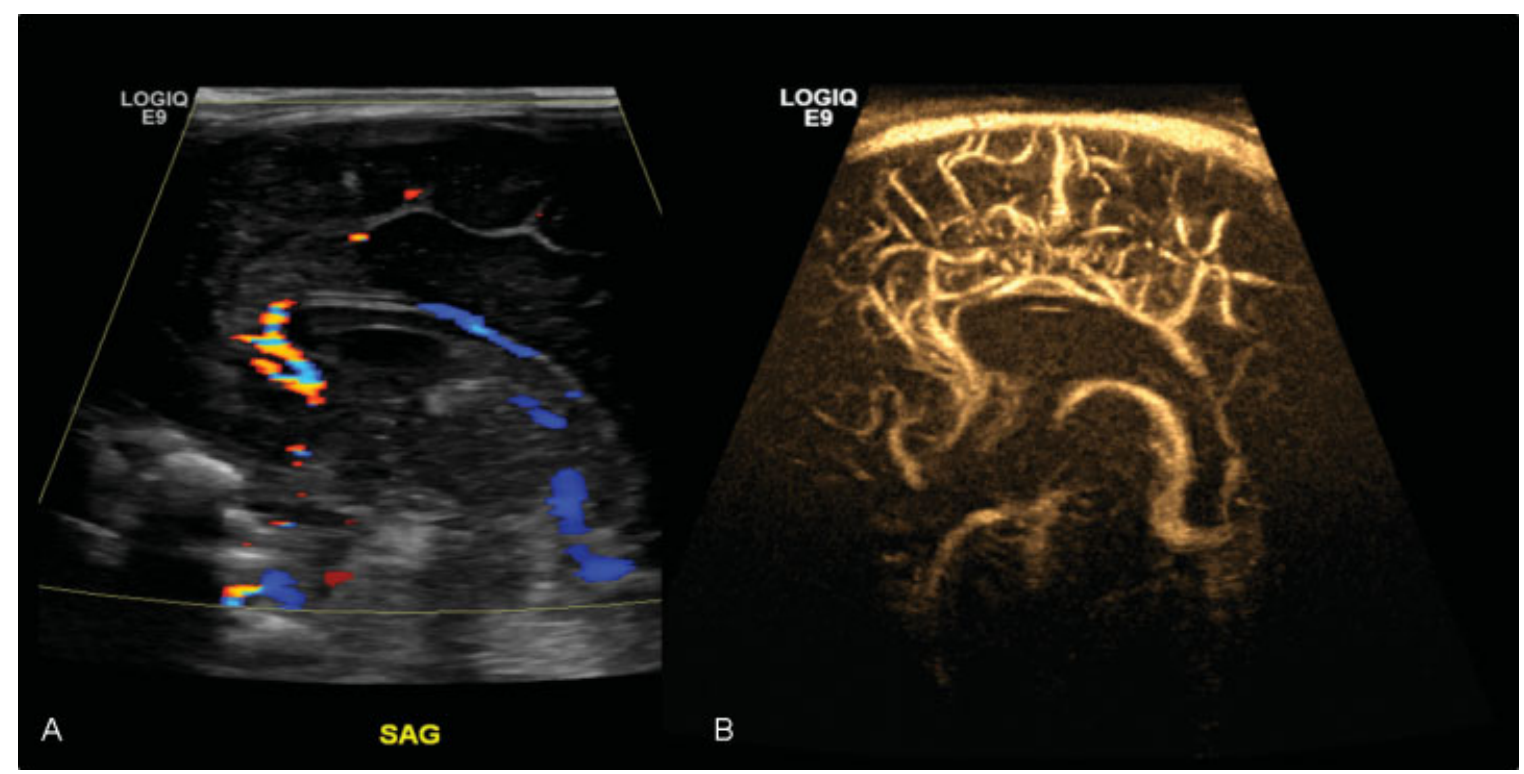

Fig. 7 (A) Color Doppler and (B) B-flow imaging in the sagittal plane through the anterior fontanel demonstrates normal vascular anatomy in the term neonate. B-flow is especially good at detecting intravascular echoes in small vessels, allowing optimal visualization of the terminal branches of the ACA and the deep venous structures in the sagittal midline. Vascular structures routinely visualized on sagittal scan include the basilar, internal carotid, and anterior cerebral arteries, as well as the internal cerebral veins, vein of Galen, the straight sinus, and the superior sagittal sinus. ACA, anterior cerebral artery. 

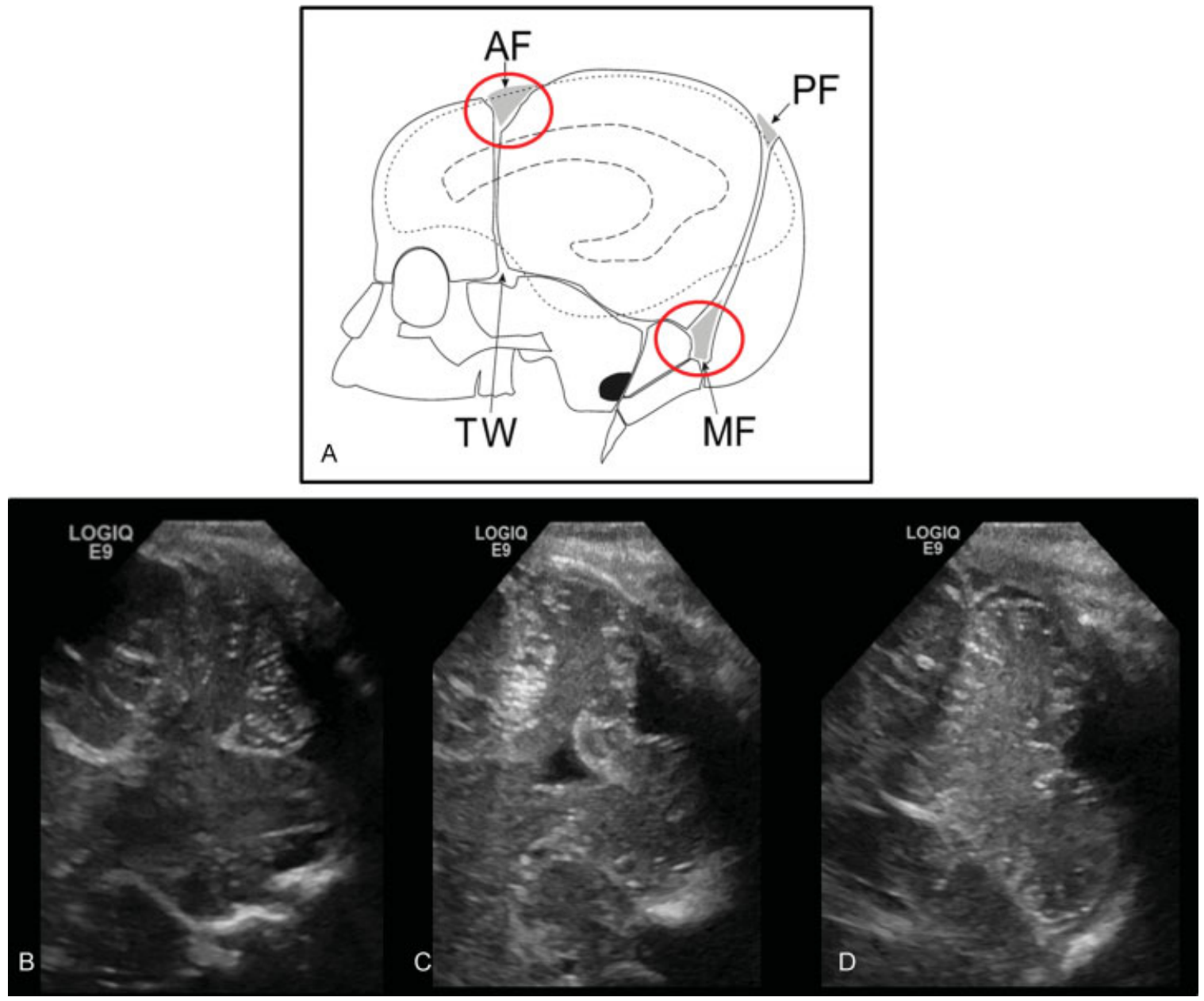

Fig. 8 (A) Possible acoustic windows for neonatal cranial ultrasound. Anterior and mastoid fontanels are the most commonly utilized in neonatal cranial ultrasound; imaging through the mastoid fontanel using sector transducer with indicator notch toward calvarial vertex allows excellent visualization of the posterior fossa structures in the coronal plane including (from anterior to posterior) (B) brain stem and cerebellar peduncles, (C) fourth ventricle and cerebellar hemispheres, and (D) cerebellar hemispheres and cerebellar vermis. ACA, anterior cerebral artery.

the evaluation of deep brain and posterior fossa structures. ${ }^{11,12}$ The mastoid fontanel is located at the junction of the squamosal, lambdoidal, and occipital sutures (-Fig. 8A) just behind the pinna of the ear. Imaging through this acoustic window with a sector or small-footprint linear transducer allows improved visualization of the posterior fossa. Images can be obtained in the coronal and axial planes through both right and left mastoid fontanels for detailed evaluation of the cerebellar hemispheres, cerebellar vermis, fourth ventricle, foramen magnum, cerebral peduncles, and brain stem (-Fig. 8B-D).

\section{Application of Advanced Techniques in Neonatal Cranial Ultrasound}

\section{Germinal Matrix-Intraventricular Hemorrhage}

The germinal matrix (GM) is a highly cellular structure of the embryologic brain from which neural and glial progenitor cells migrate during brain development. The GM is centered in the subependymal caudothalamic groove at the base of the lateral ventricles. The GM reaches maximal volume at 24 to 26 weeks' gestation and then regresses over the next
10 weeks with only scattered areas of residual GM seen in the caudothalamic groove at 36 to 38 weeks' gestation. ${ }^{2}$

The GM is highly vascular, supplied by a network of fragile capillaries originating from the recurrent artery of Hubner and from penetrating striate and anterior choroidal arteries. $^{2,11}$ Fluctuations in systemic blood pressure and alterations in oxygenation, especially in the presence of increased venous pressure, lead to rupture of the fragile GM capillaries with resultant hemorrhage. These physiologic changes are most profound around the time of delivery and early neonatal life with up to $90 \%$ of GM hemorrhage occurring within the first 4 days. ${ }^{13}$

GM hemorrhage has traditionally been classified by the anatomic location of blood products according to the Papile grading system. ${ }^{2,14}$ The use of high-frequency linear transducers in neonatal cranial ultrasound has greatly improved visualization of these hemorrhages with increased accuracy in grading.

Acute blood appears hyperechoic to the normal choroid plexus; however, as blood ages, it becomes more heterogeneous and may appear iso- to hypoechoic to the choroid plexus and thus be more difficult to detect on ultrasound. 


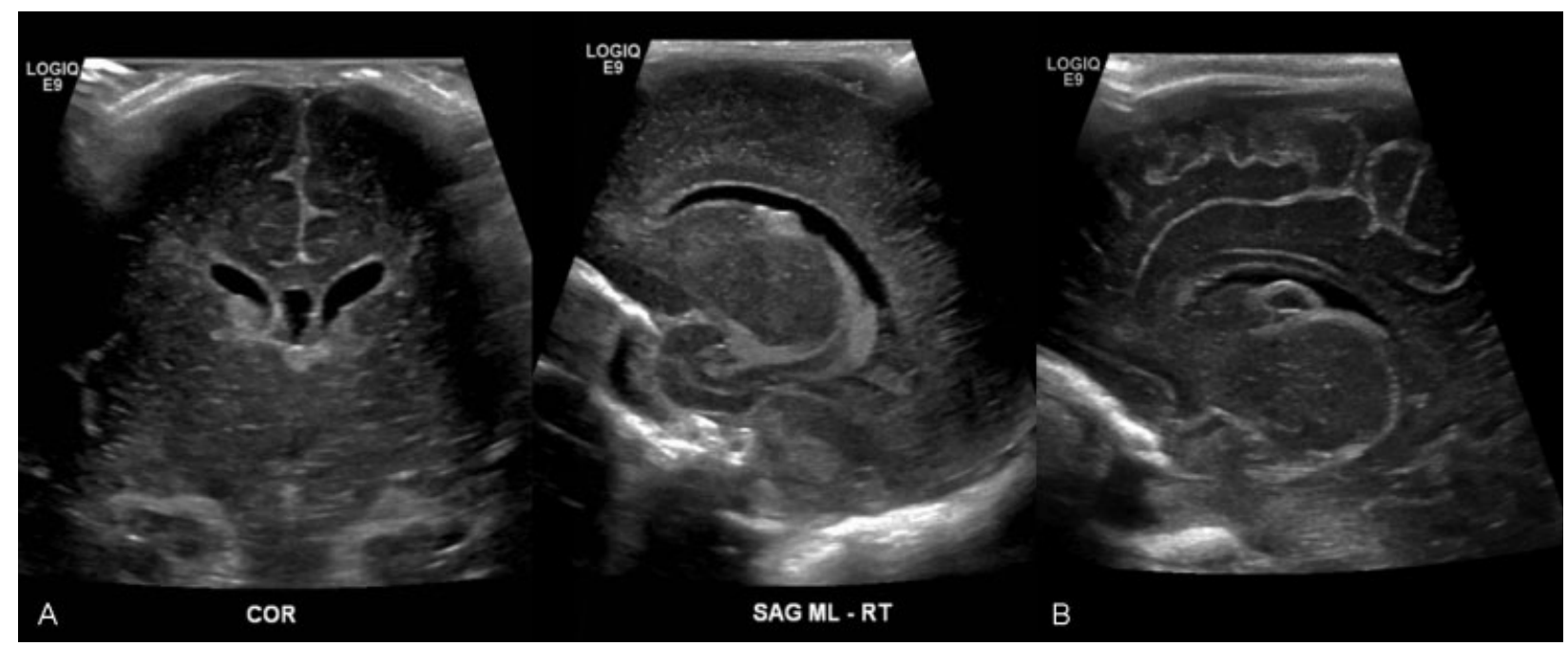

Fig. 9 Grade $1 \mathrm{GM}$ hemorrhage, confined to the germinal matrix. (A) Coronal and sagittal imaging with a 9-MHz linear transducer allows excellent visualization of acute, echogenic hemorrhage effacing the normal V-shaped caudothalamic groove, with no echogenic intraventricular blood products; (B) resolving hemorrhage at the caudothalamic groove appears hypoechoic. ACA, anterior cerebral artery; GM, germinal matrix.

Grade 1 hemorrhage is localized to the GM and is bounded by the overlying ependyma. On ultrasound, Grade 1 hemorrhage is seen as an echogenic focus that effaces the normal $V$ shaped caudothalamic groove, bulging upward into the ventricle with no echogenic intraventricular blood products. Grade I hemorrhages account for 75 to $80 \%$ of all GM-IVH (-Fig. 9).

Grade 2 hemorrhage begins in the GM and extends through the overlying ependyma into the lateral ventricle. By definition, Grade 2 GM-IVH echogenic blood products fill less than $50 \%$ of the lateral ventricle and there is no associated ventricular dilation (- Fig. 10). Hemorrhage abutting the choroid plexus can be distinguished by its heterogeneity and "lumpy-bumpy" contour. Echogenicity in the anterior horns of the lateral ventricles should be recognized as evidence of intraventricular extension as choroid plexus does not extend anterior to the foramen of Monro. Similarly, echogenicity layering in the occipital horns should be recognized as IVH as the choroid plexus does not extend this far posterior. $^{2}$

Grade 3 hemorrhage involves a larger amount of intraventricular blood (filling more than $50 \%$ of lateral ventricle) and is associated with ventricular dilation (-Fig. 11). The third and fourth ventricles should be carefully evaluated for echogenic blood products. The third ventricle is seen to maximal advantage in the coronal and sagittal imaging planes using

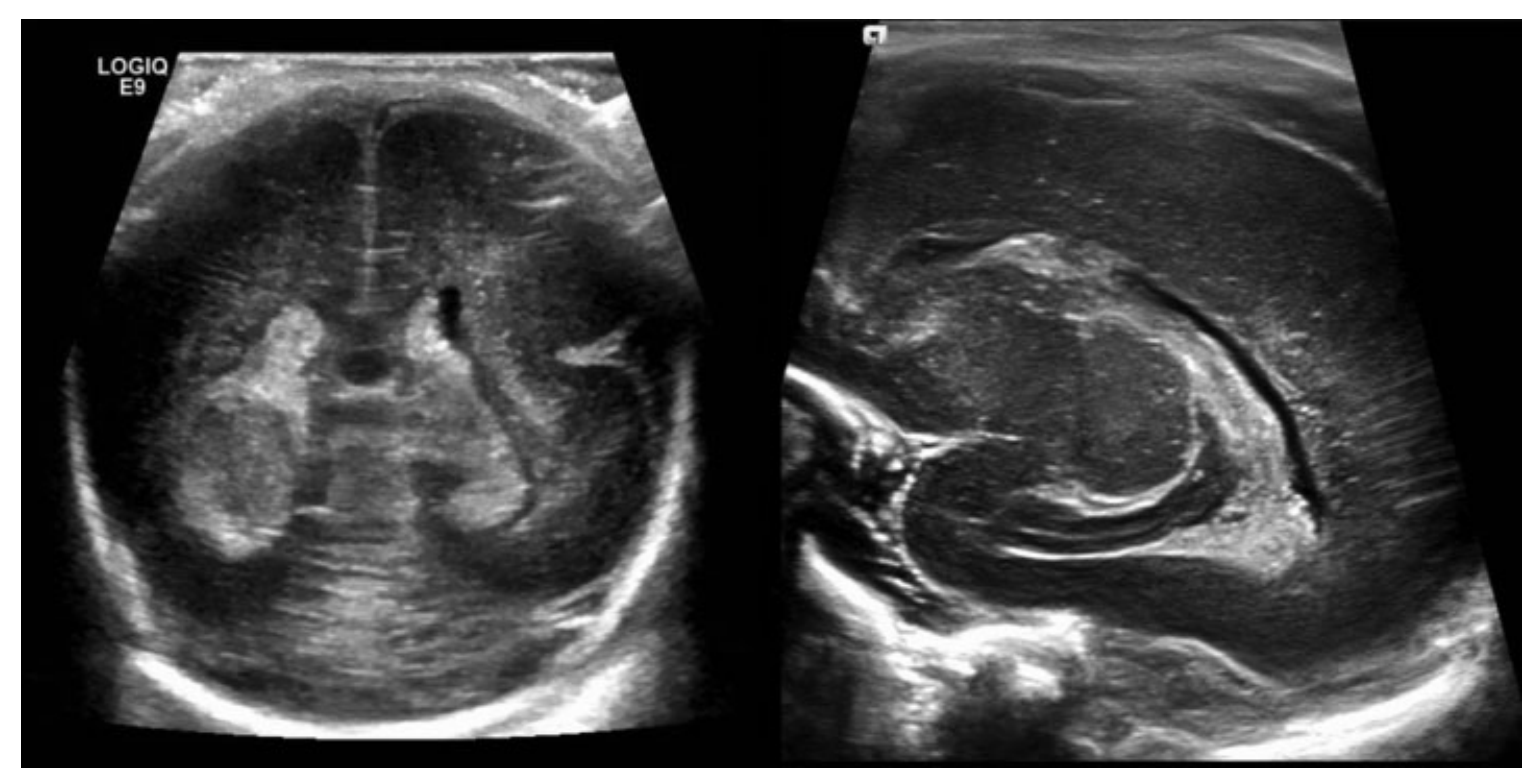

Fig. 10 Grade $2 \mathrm{GM}$ hemorrhage/IVH. Coronal and sagittal imaging with a 9-MHz linear transducer allows excellent visualization of echogenic hemorrhage extending into the right lateral ventricle with no associated ventricular enlargement. Note echogenic material in the lateral ventricle anterior to the caudothalamic groove and in the occipital horns. ACA, anterior cerebral artery; GM, germinal matrix; IVH, intraventricular hemorrhage. 


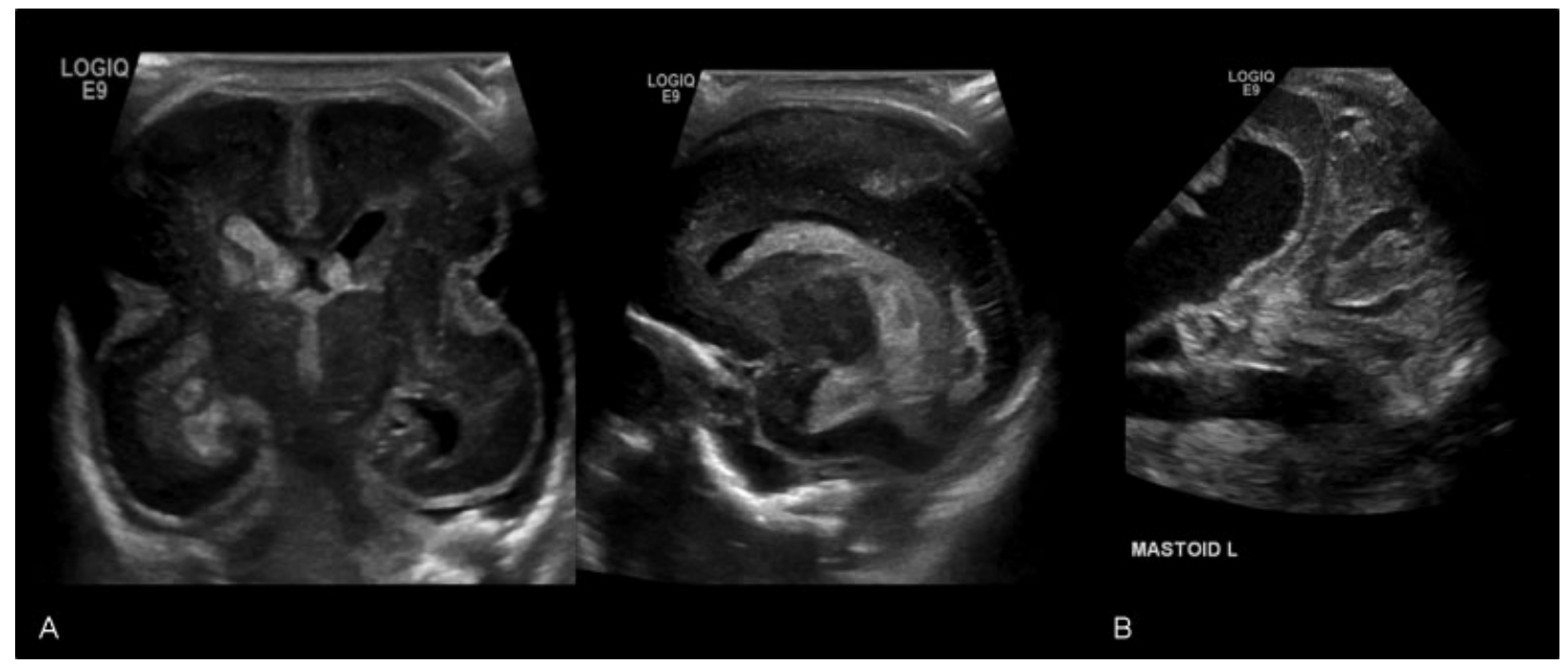

Fig. 11 Grade 3 GM hemorrhage/IVH. (A) Coronal and sagittal imaging with a 9-MHz linear transducer provides optimal visualization of acute hemorrhage in the right lateral ventricle with associated ventricle enlargement plus echogenic acute hemorrhage in the third ventricle. Note the extremely premature sulcation pattern in the cerebral hemispheres in this premature infant born at 26 weeks' gestation. (B) Transmastoid images in a different infant demonstrates echogenic blood products in the fourth ventricle. GM, germinal matrix; IVH, intraventricular hemorrhage.

high-frequency linear transducer. Transmastoid views of the posterior fossa allow for improved visualization of hemorrhage within the fourth ventricle (-Fig. 11B). ${ }^{2,12}$

Grade 4 hemorrhage was classically defined by Papile et al as "extension of intraventricular blood into the surrounding brain parenchyma" described as a fan-shaped region of increased echogenicity in the periventricular white matter associated with IVH. However, as clinical understanding and imaging technology has advanced, this finding is now recog- nized as periventricular hemorrhagic infarct (PVHI). $2,8,9,13$ This injury is thought to result from compression or obstruction of terminal veins that drain the periventricular white matter secondary to mass effect from ipsilateral GM hemorrhage. ${ }^{2,8}$ This is the least common type of GM-IVH, occurring in approximately $5 \%$ of premature infants.

On ultrasound, Grade $4 \mathrm{GM}-\mathrm{IVH} / \mathrm{PVHI}$ appears as a region of increased echogenicity in the periventricular white matter along the atrium of the lateral ventricle (-Fig. 12B) and is

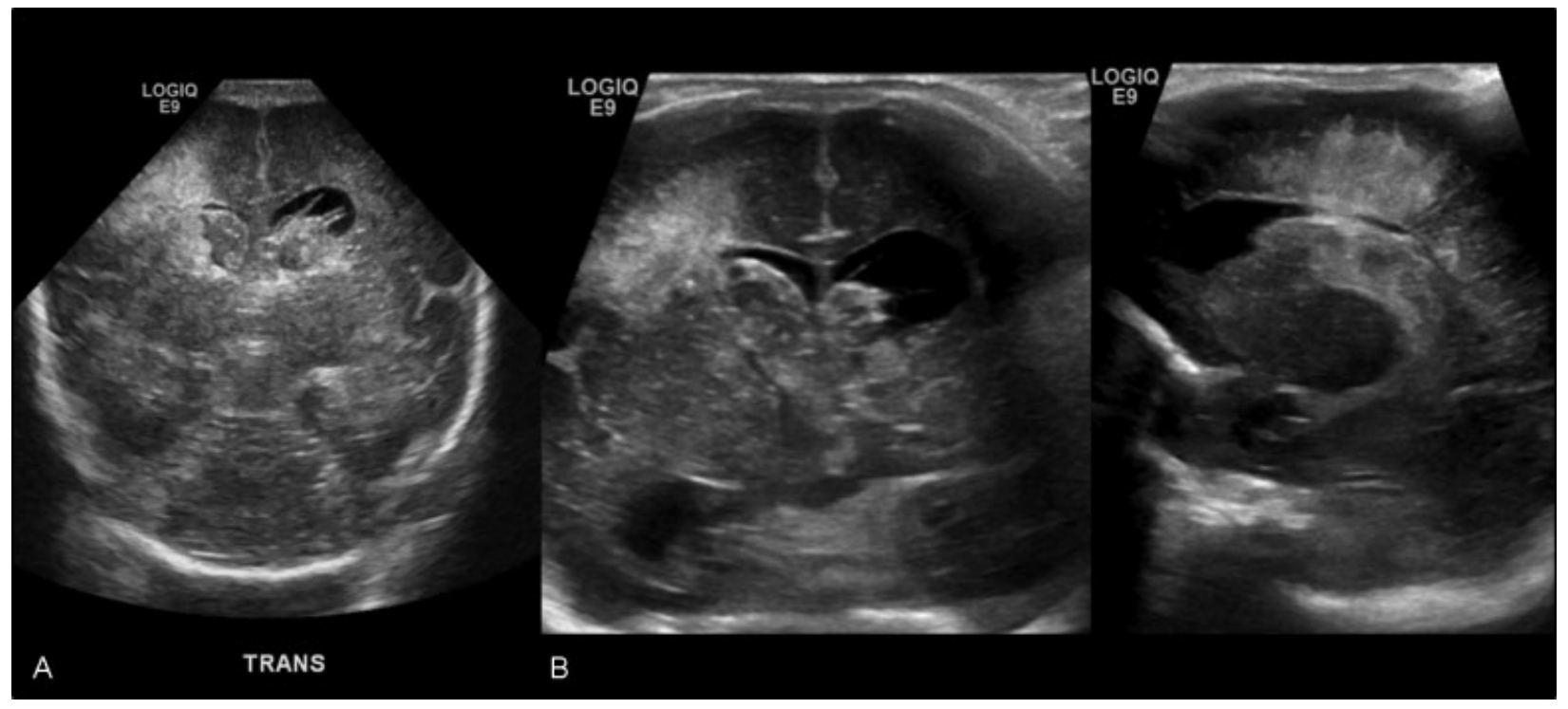

Fig. 12 Grade 4 GM-IVH/PVHI. (A) Coronal ultrasound images with sector transducer; (B) coronal and sagittal ultrasound images in the same patient with $9-\mathrm{MHz}$ linear transducer allows more detailed evaluation of the lateral ventricles and brain parenchyma, highlighting echogenic intraventricular blood products and echogenic hemorrhage in the periventricular white matter with preservation of the lateral ventricle wall. $\mathrm{GM}$, germinal matrix; IVH, intraventricular hemorrhage; PVHI, periventricular hemorrhagic infarct. 
always associated with an ipsilateral GM-IVH. Size of infarct varies markedly depending on the area of drainage of the occluded vein and amount of collateral venous drainage present. Color Doppler may show lack of flow in the terminal vein.

A scoring system has been proposed for PVHI at the time of initial diagnosis that has been shown to correlate with clinical risk factors and outcomes (-Fig. 13). 2,13 This system assigns a score of 0 or 1 in three categories of findings on ultrasound in PVHI. First, extent of periventricular white matter involvement is scored. To do this, the cerebral hemisphere is divided into five territories in the sagittal plane (-Fig. 13B). A territory is considered "involved" when internal echogenicity measures $\geq 5 \mathrm{~mm}$. If only a single territory is involved, PHVI is scored as "localized." If two or more territories are involved, PVHI is scored as "extensive." Second, PVHI is scored as unilateral or bilateral. Finally, associated midline shift is scored as absent or present. The score in each category is then added together. In the study reported by Bassan et al, the higher the total score of PVHI injury, the more likely infants were to have poor longterm outcomes. $^{15}$

Once the initial diagnosis of GM-IVH is made, follow-up ultrasound is typically performed 1 week later to evaluate for interval change. Serial ultrasounds may be performed until hemorrhage resolves. Resolving intraventricular blood products become more heterogeneous as they undergo cystic transformation and liquefaction. ${ }^{2,8,9}$ These changes are usually not encountered until the second or third week following acute hemorrhage.

Small Grade 1 GM-IVH commonly resolves without any residual abnormality on ultrasound. Approximately $30 \%$ of premature infants with Grades 2 to 4 GM-IVH will develop posthemorrhagic ventricular dilation (-Fig. 14), thought secondary to obliterative arachnoiditis and/or obstruction in the basilar cistern or fourth ventricle outflow tract that blocks normal flow of CSF. ${ }^{13}$ Pulsed wave Doppler evaluation of the ACA in these infants, discussed in detail later, allows for early identification of increased ICP that may require ventricular shunting (- Fig. 15). ${ }^{7}$

In the setting of Grade $4 \mathrm{GM}-\mathrm{IVH} / \mathrm{PVHI}$, as periventricular hemorrhage slowly resolves, the underlying brain parenchyma may undergo liquefaction resulting in cyst formation and volume loss. The adjacent ventricle may expand into the affected region, ex vacuo dilation with porencephalic cyst formation (-Fig. 16). These findings are seen to best advantage on ultrasound imaging performed with a high-frequency linear transducer. ${ }^{2,3}$

Grade 3 GM-IVH and Grade 4 GM-IVH/PVHI are associated with up to $50 \%$ probability of neurologic morbidity including cerebral palsy and developmental delay. In contrast, it appears that the presence of a Grade 1 or Grade 2 GM-IVH does not measurably alter overall risk of poor neurologic outcome in premature infants. ${ }^{13}$

\section{Hypoxic-Ischemic Injury}

HII affects both term and preterm infants in the clinical setting of hypoxemia and altered cerebral blood flow. In infants, hypoxemia may result secondary to several factors including perinatal asphyxia, hypercapnia (retention of $\mathrm{CO}_{2}$ ) in the setting of lung disease, complex inflammatory processes, and congenital heart disease. ${ }^{2,8}$ Alterations in cerebral blood flow may occur in the setting of both hypotension and hypertension. In addition, infants have limited capacity for cerebral autoregulation, the process by which the brain

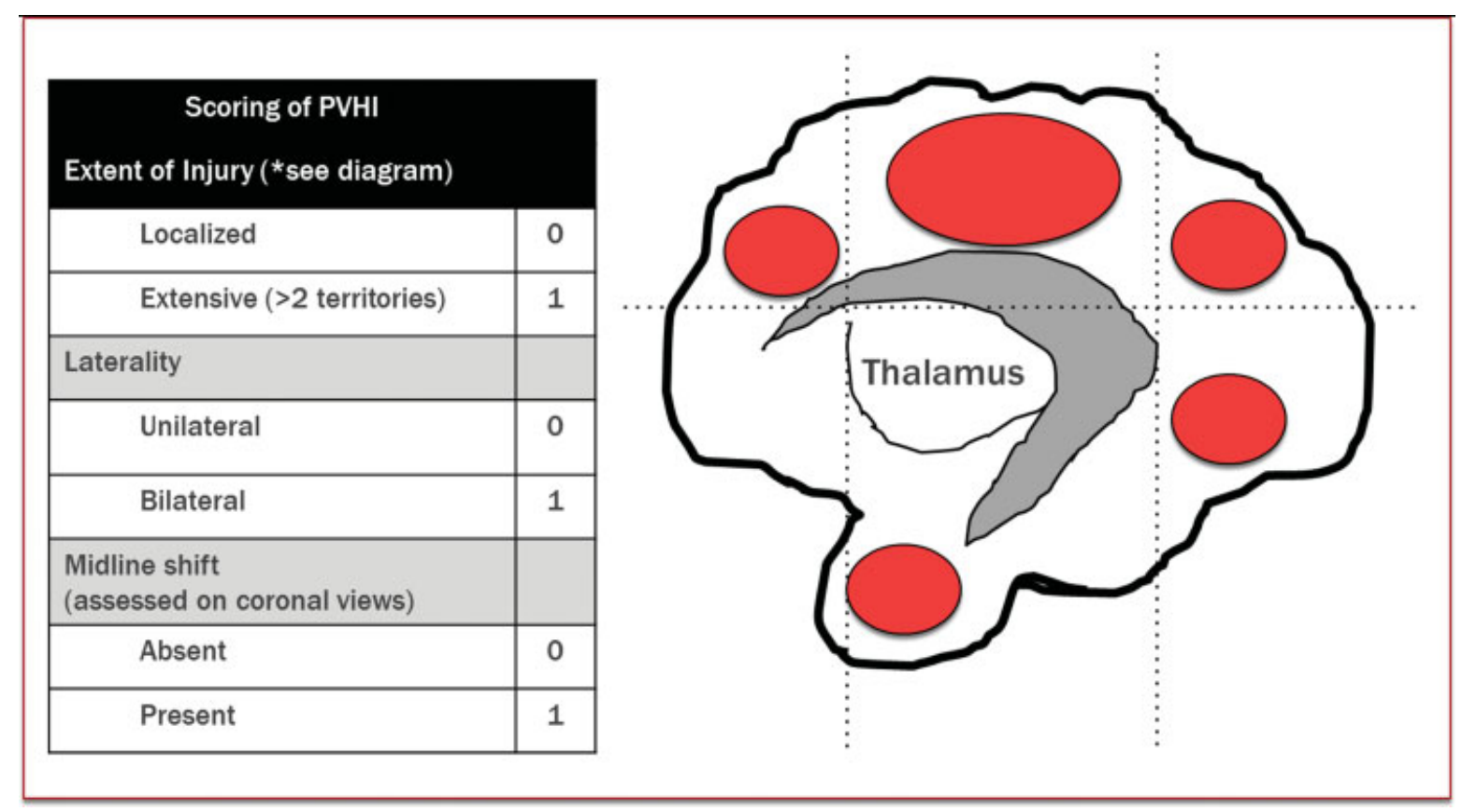

Fig. 13 Scoring system of PVHI proposed by Bassan et al. (A) Categories and scoring system and (B) cerebral territories for scoring of periventricular hemorrhagic infarct. PVHI, periventricular hemorrhagic infarct. 


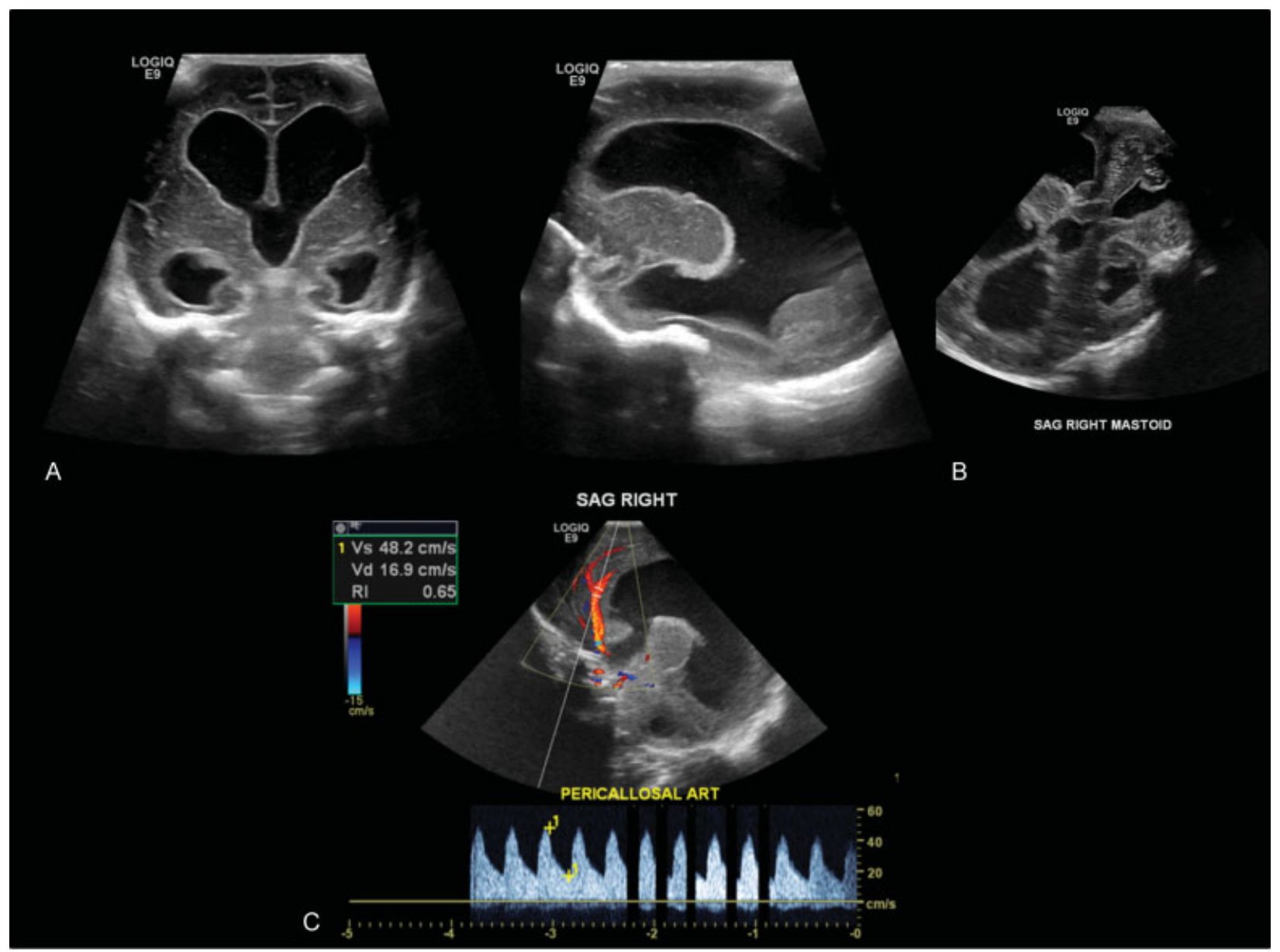

Fig. 14 Complications of GM-IVH/PVHI. (A) Coronal and sagittal ultrasound images with 9-MHz linear transducer in infant with a history of Grade $3 \mathrm{GM}-\mathrm{IVH}$ demonstrate severe posthemorrhagic ventricular dilation, (B) transmastoid view with 7-MHz sector transducer demonstrates dilation of the third and fourth ventricles, and (C) pulsed wave Doppler evaluation of the ACA demonstrates normal RI in the setting of normal ICP. ACA, anterior cerebral artery; GM, germinal matrix; ICP, intracranial pressures; IVH, intraventricular hemorrhage; PVHI, periventricular hemorrhagic infarct; RI, resistive index.

maintains a relatively constant cerebral blood flow, which puts them at higher risk for HII.

The pattern of brain injury in HII depends on severity and duration of hypoxic-ischemic event as well as degree of brain maturity at the time of injury. ${ }^{2}$

Injury to the brain is thought to be a two-step process occurring during ischemia and subsequent reperfusion. During episodes of hypoxia and altered cerebral blood flow, the brain compensates by shunting blood to the most metabolically active parts of the brain and to centers most crucial for maintenance of basal function including the brain stem, basal ganglia, and cerebellum. Thus, short episodes of hypoxia and ischemia will predominantly affect the more peripheral "watershed zones" of cerebral perfusion. ${ }^{2,13}$ However, more severe episodes lasting $>15$ to 25 minutes will result in injury to the deep gray matter. Reperfusion with the resolution of hypoxia and ischemia results in increased flow to the affected areas of the brain, predisposing to hemorrhage.

Before 32 weeks' gestation, brain circulation is significantly different than that seen in late gestation or neonatal life. The preterm brain parenchyma is supplied by a network of penetrating arteries extending from the pial surface of the brain and deep intracerebral arteries. This results in relatively poor vascularization of the periventricular white matter, the preterm "watershed zone." The areas, most prone to injury lie along the dorsal and lateral margins of the lateral ventricles in the region of the centrum semiovale and the optic and acoustic radiations. ${ }^{2,16}$

The "watershed zones" gradually move more peripherally with continued cerebral vascular ingrowth and by 38 weeks gestational age are seen in the mature and more familiar ACA/MCA and MCA/PCA distribution. Thus, mild-to-moderate $\mathrm{HII}$ at term gestation results in injury of the basal ganglia and peripheral cortex with relative sparing of the periventricular white matter.

When performed with high-frequency linear transducers, attention to detail, and careful technique, ultrasound is a powerful diagnostic tool that can be used at the bedside in the detection of white matter injury in preterm and term infants. In fact, a study by Epelman et al demonstrated that normal cranial ultrasound in the setting of HII has a negative 


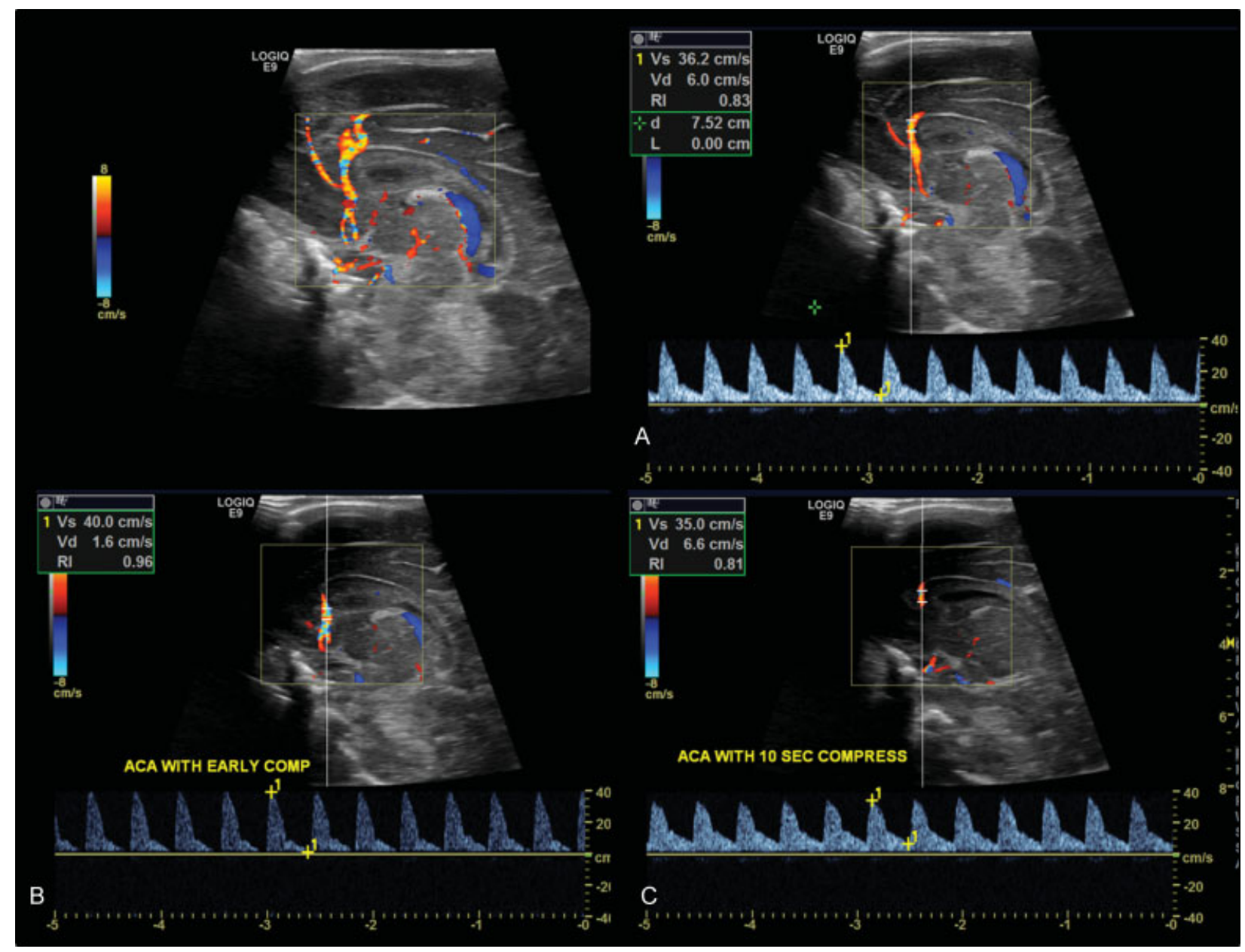

Fig. 15 Normal pulsed wave Doppler of ACA in term infant. (A) Normal ACA RI in preterm infant $(<0.85)$, (B) transient increase in ACA RI with application of gentle anterior fontanel pressure, and (C) normalization of ACA RI after 10 seconds of anterior fontanel pressure, indicating normal cerebral autoregulation in the setting of normal ICP. ACA, anterior cerebral artery; RI, resistive index.

predictive value of nearly $100 \%$ and may preclude the need for neonatal brain MRI. ${ }^{13}$

In preterm infants, ultrasound may demonstrate early changes from HII as an ill-defined and poorly marginated echogenicities in the periventricular white matter. Unlike PVHI, HII is more often bilateral and symmetric ( - Fig. 17) ${ }^{2,8,17}$ It should be noted that severe HII may be complicated by secondary hemorrhage and can be extremely difficult to differentiate from PVHI.

Follow-up ultrasound shows that most of these lesions resolve. Periventricular echogenicity lasting beyond the first week of life are more likely to be clinically significant. Severely injured periventricular brain parenchyma can undergo cystic necrosis known as periventricular leukomalacia (PVL). Specific patterns of echogenicity and cystic necrosis in PVL have been classified by de Vries et al (-Fig. 18) ${ }^{18}$

In term infants, mild-to-moderate HII results in diffuse injury to the watershed zones of the cerebral hemispheres. On ultrasound, this is seen as diffusely increased echogenicity of the white matter resulting in accentuated graywhite matter differentiation (-Fig. 19). Imaging with a high-frequency transducer allows for excellent visualization of the cerebral cortex and increases sensitivity of ultrasound in HII. However, findings may be subtle and require specific attention by the interpreting radiologist. ${ }^{2,19,20}$ Signs of diffuse cerebral edema include slit-like appearance of the lateral ventricles and effacement of the extra-axial spaces.

Doppler imaging can be used to further evaluate cerebral blood flow in the setting of HII. Acutely, higher end-diastolic flow velocities due to vasodilation in the setting of increased vascular demand, result in decreased RI ( - Fig. 5A) ${ }^{2,7}$ Multiple studies have shown that an RI value below 0.55 measured in the ACA in the first 24 to 72 hours of life following HII correlates with a poor neurodevelopment prognosis., ${ }^{717}$ Later in the course of brain injury, as cerebral edema increases, increased ICP results in elevated RI. ${ }^{2,7}$

In severe HII injury to deep brain structures is seen on ultrasound as diffusely increased echogenicity in the basal ganglia and thalami, well visualized using linear array transducers. 


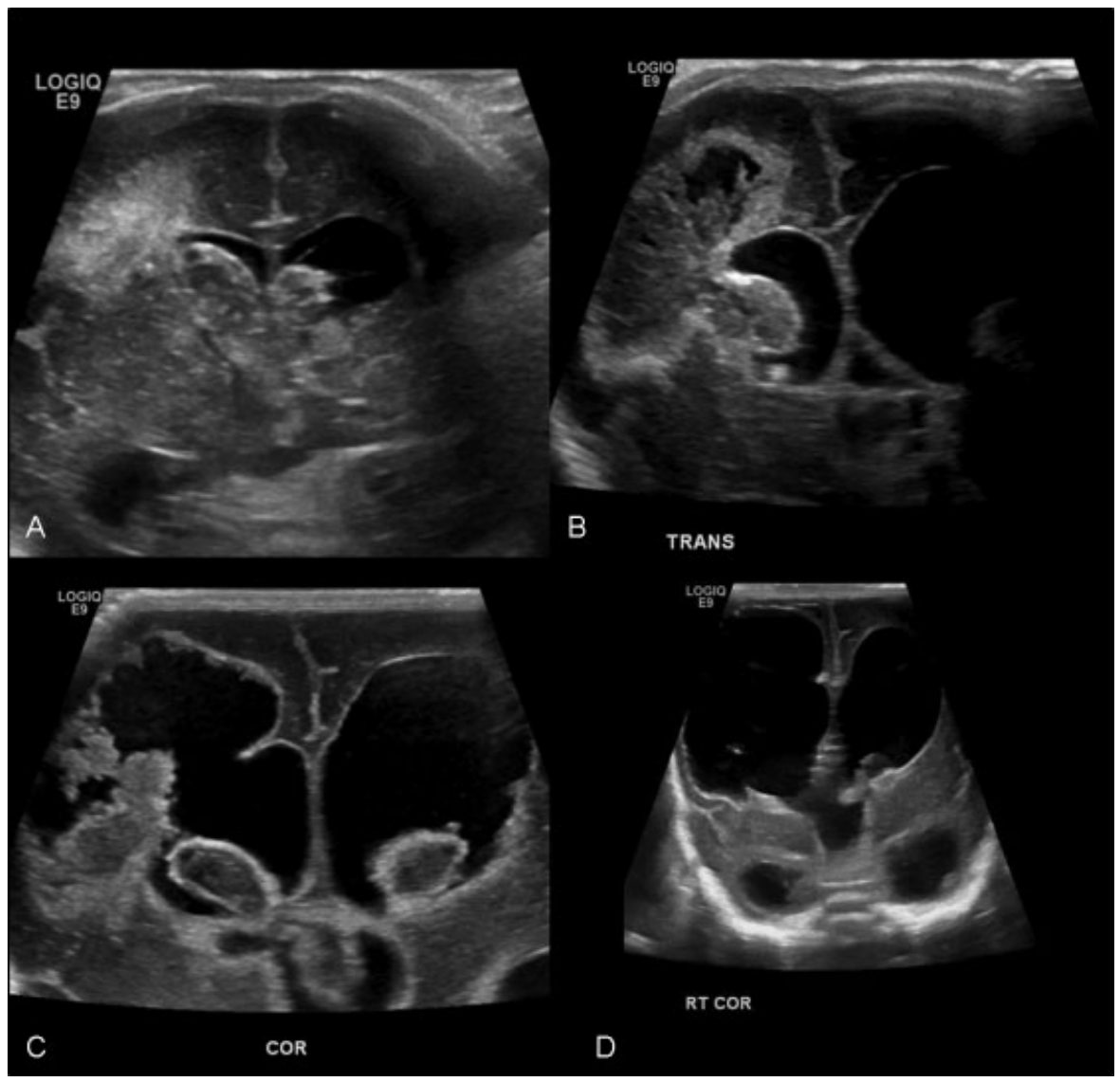

Fig. 16 Complications of Grade $4 \mathrm{GM}-\mathrm{IVH} / \mathrm{PVHI}$. Serial ultrasound imaging performed with 9-MHz linear transducer of preterm infant with Grade $4 \mathrm{GM}-\mathrm{IVH} / \mathrm{PVHI}$ at (A) first week of life, (B) 4 weeks, (C) 8 weeks, and (D) 12 weeks demonstrates progressive cystic change in the periventricular white matter with eventual formation of a large porencephalic cyst and ex vacuo dilation of the right lateral ventricle. ACA, anterior cerebral artery; GM, germinal matrix; IVH, intraventricular hemorrhage; PVHI, periventricular hemorrhagic infarct.

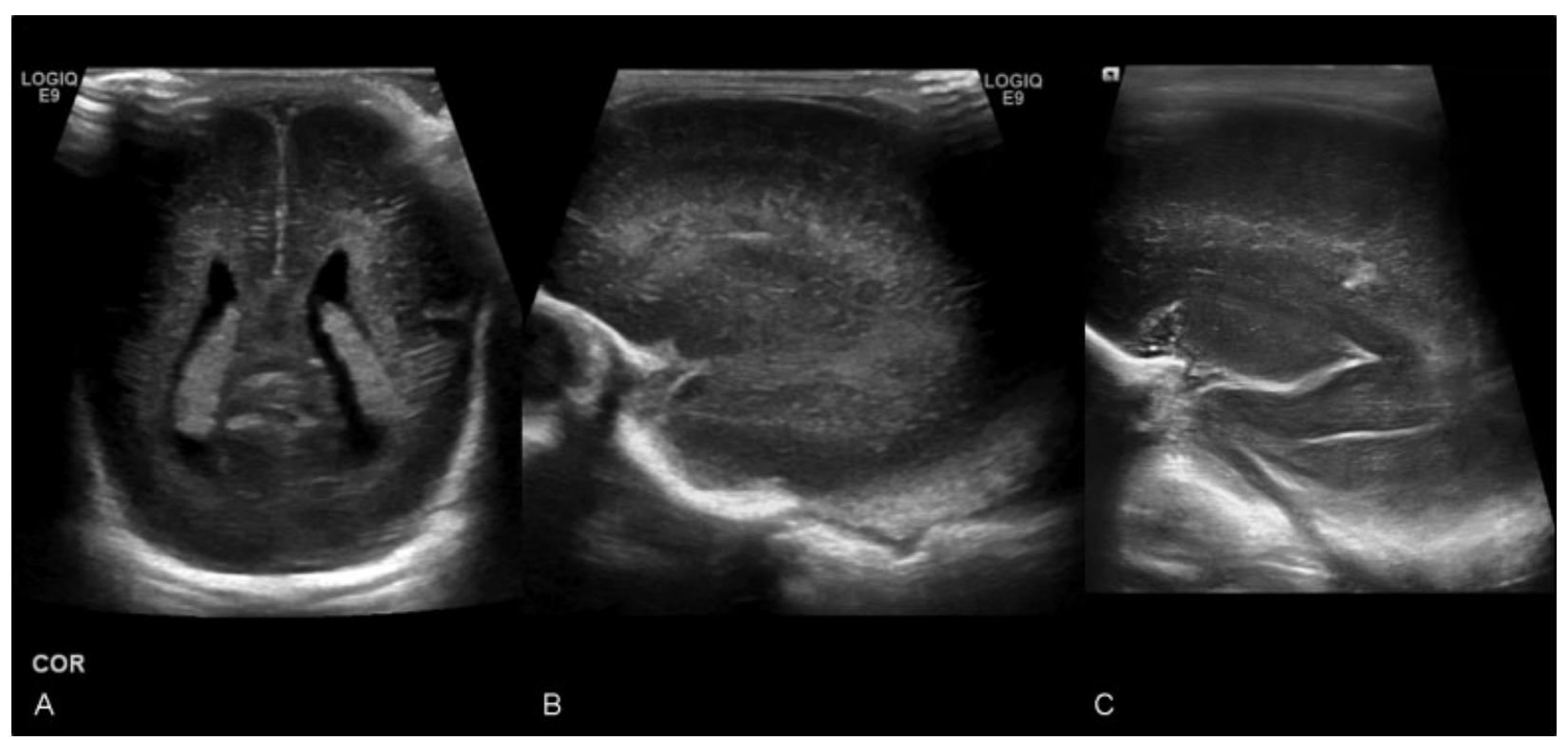

Fig. $17 \mathrm{HII}$ in preterm infant. Ultrasound imaging with 9-MHz linear transducer in the (A) coronal, (B) right parasagittal, and (C) left parasagittal planes demonstrates increased echogenicity in the periventricular white matter in "watershed" distribution consistent with HII. Note normal appearance of the lateral ventricles with no intraventricular hemorrhage to distinguish this finding from Grade 4 GM-PVHI. GM, germinal matrix; HII, hypoxic-ischemic injury; PVHI, periventricular hemorrhagic infarct. 


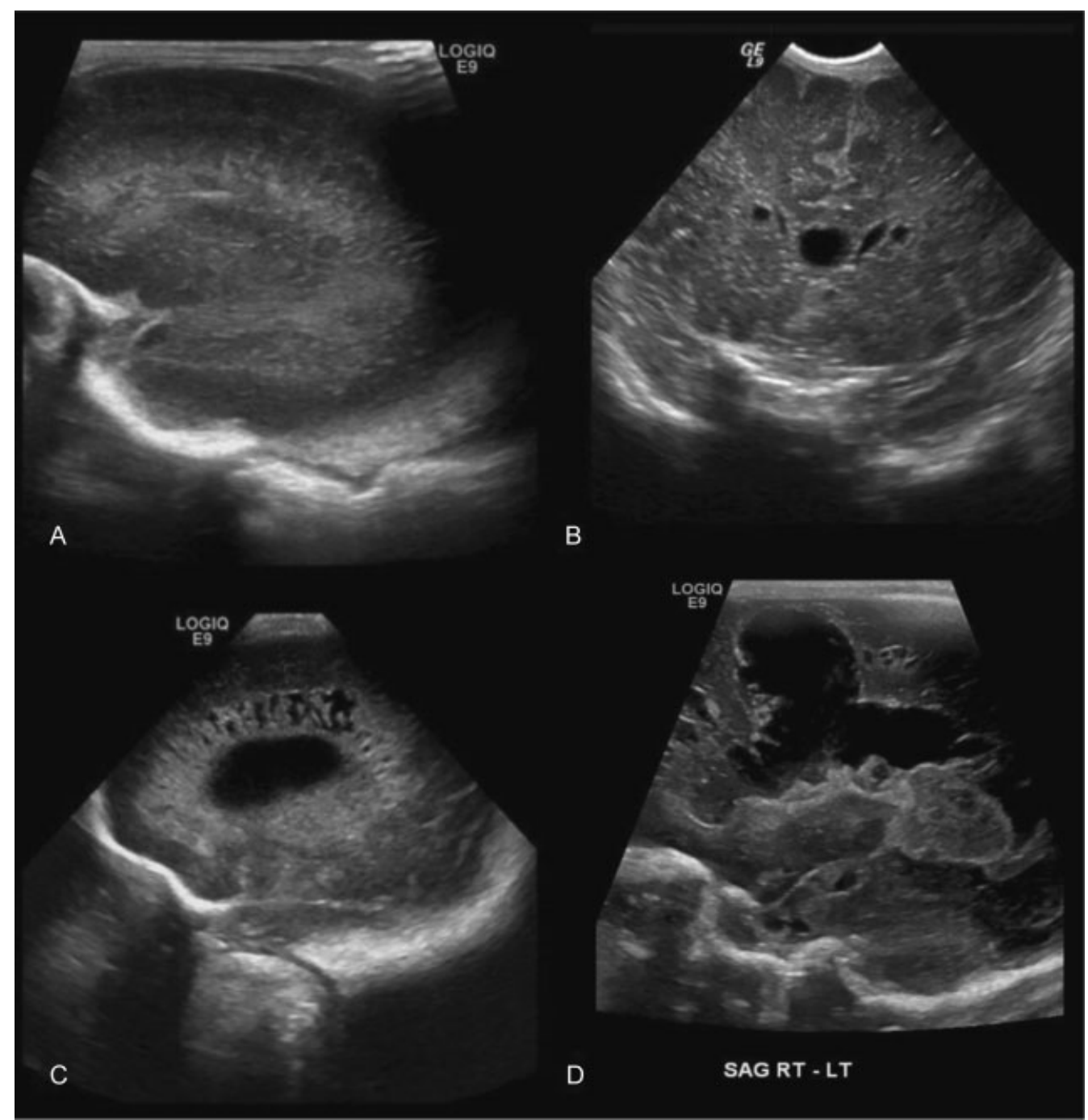

Fig. 18 Patterns of PVL following HII. (A) Grade 1 PVL, periventricular echogenicity persisting more than 7 days, (B) Grade 2 PVL, small periventricular cysts, (C) Grade 3 PVL, extensive periventricular cysts, and (d) Grade 4 PVL, extensive periventricular and subcortical cysts. Note that parenchymal cystic change in PVL results in smaller cysts than those seen in preterm PVHI. HII, hypoxic-ischemic injury; PVHI, periventricular hemorrhagic infarct; PVL, periventricular leukomalacia.

\section{Cerebellar Injury}

Injury to the developing cerebellum has been described as an additional complication of prematurity that can occur in the setting of HII, most commonly in extreme prematurity and ELBW in the setting of hypotension with major impact on neurodevelopmental outcome. ${ }^{16}$

Infarct is most common at the watershed distribution between superior cerebellar artery and PICA and may have hemorrhagic conversion. This finding may be extremely challenging to detect on standard imaging through the anterior fontanel but may be easily identified with imaging through the mastoid fontanel (- Fig. 20). ${ }^{12}$

\section{Ventriculomegaly}

Ventricular enlargement may be due to multiple etiologies in the neonatal period. When detected on prenatal ultrasound, ventriculomegaly is more likely due to congenital CNS anomaly. Ventricular enlargement may also occur secondary to white matter atrophy following HII. When this occurs, the ventricles slowly enlarge over time and eventually stabilize. Obstructive hydrocephalus occurs when there is obstruction of CSF drainage pathways at the level of the aqueduct, fourth ventricular outflow pathways, or arachnoid granulations. When this occurs in the setting of GM/IVH, it may result in rapid enlargement of the ventricles on ultrasound (-Fig. 14A, B). ${ }^{2,8,9}$

Distinguishing between obstructive versus nonobstructive hydrocephalus is clinically important, as the former necessitates neurosurgical consultation and potential intervention. Serial ultrasound with measurement of ventricular size can identify rate and extent of ventricular enlargement.

On postnatal cranial ultrasound, the biventricular, bifrontal diameter of the lateral ventricles is measured at the level of the foramen of Monro in the coronal plane as the distance between the lateral walls of both lateral ventricles (-Fig. 21). ${ }^{2}$ Transverse oblique measurements of the 


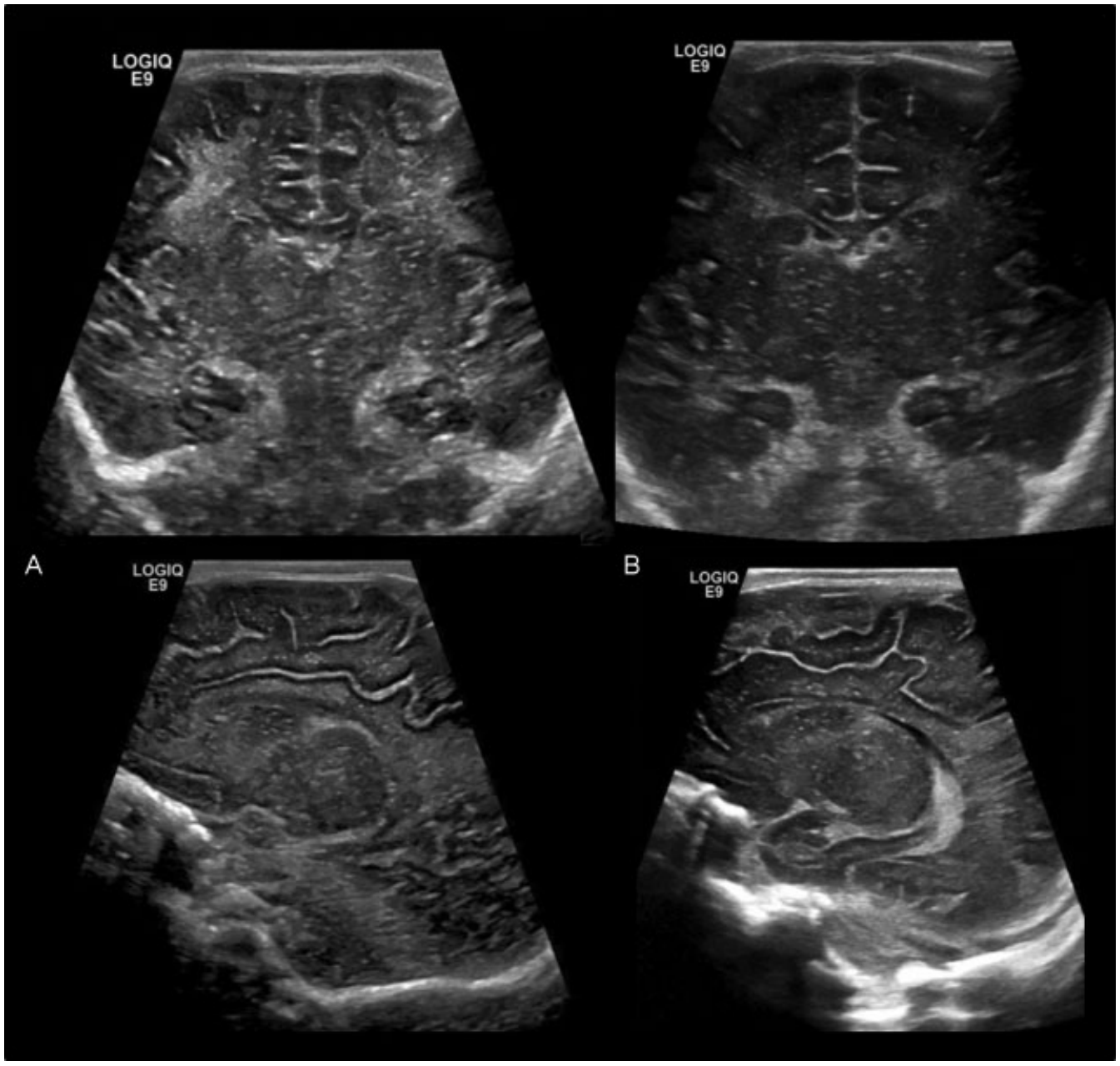

Fig. $19 \mathrm{HII}$ in term infant. Ultrasound imaging with 9-MHz linear transducer in the (A) coronal and parasagittal planes demonstrates diffusely increased echogenicity of the white matter resulting in accentuated gray-white matter differentiation and (B) normal gray-white matter differentiation in term infant provided for comparison. HII, hypoxic-ischemic injury.

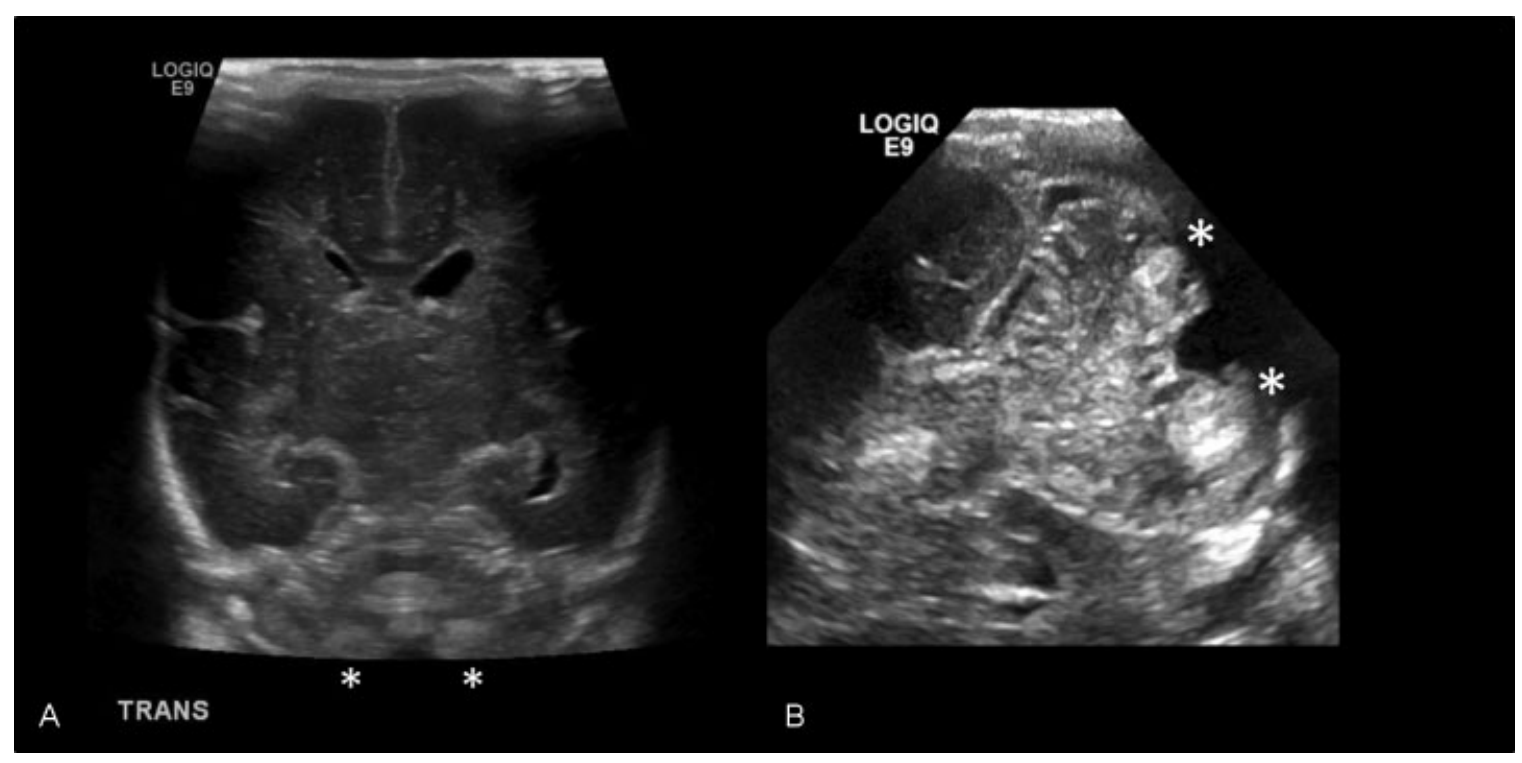

Fig. 20 Cerebellar injury. (A) Abnormal echogenicity at the base of cerebellum $\left({ }^{*}\right)$. This finding is challenging to identify on imaging via the anterior fontanel, even with use of high-frequency linear transducer. (B) Transmastoid images performed with 7-MHz sector transducer better demonstrate abnormal echogenicity at the base of both cerebellar hemispheres $\left({ }^{*}\right)$ indicative of hemorrhagic or ischemic injury. 


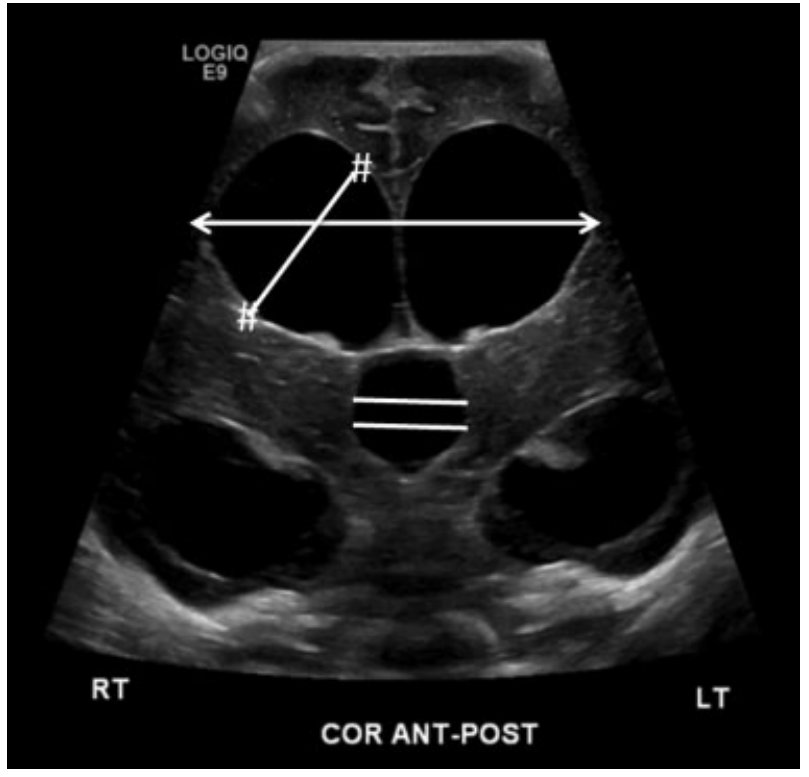

Fig. 21 Measurement of the lateral ventricles in the coronal plane at the level of the foramen of Monro in infant with ventriculomegaly including biventricular, bifrontal diameter $(\longleftarrow \rightarrow)$, transverse oblique diameter (\#-\#), and transverse diameter of the third ventricle (===). individual lateral ventricles and transverse measurements of the third ventricle are also made at the same level (-Fig. 21) ${ }^{2}$

Pulsed-wave Doppler measurement of RI in the ACA in patients with ventriculomegaly may be used to assess for increased ICP in the setting of obstructive hydrocephalus. ${ }^{2,7}$ As ICP rises, extravascular pressure results in increased vascular resistance, decreased end-diastolic flow, and increased RI ( - Fig. 14C). When extravascular pressure exceeds systemic intravascular pressure, end-diastolic flow may be reversed.

Sensitivity of pulsed wave Doppler evaluation for elevated ICP is improved with application of compression on the anterior fontanel. ${ }^{7}$ Gentle downward pressure applied with ultrasound transducer to the anterior fontanel will result in mild increase in ICP. Pulsed wave Doppler of the ACA during and immediately after pressure application will demonstrate transiently decreased end-diastolic flow with increased RI. In the normal infant, cerebral autoregulation will correct for the slight increase in ICP and flow within the ACA will normalize within 5 seconds with return to a normal RI (-Fig. 15).

When ICP is already increased, as in severe hydrocephalus, compression of the anterior fontanel will more drastically

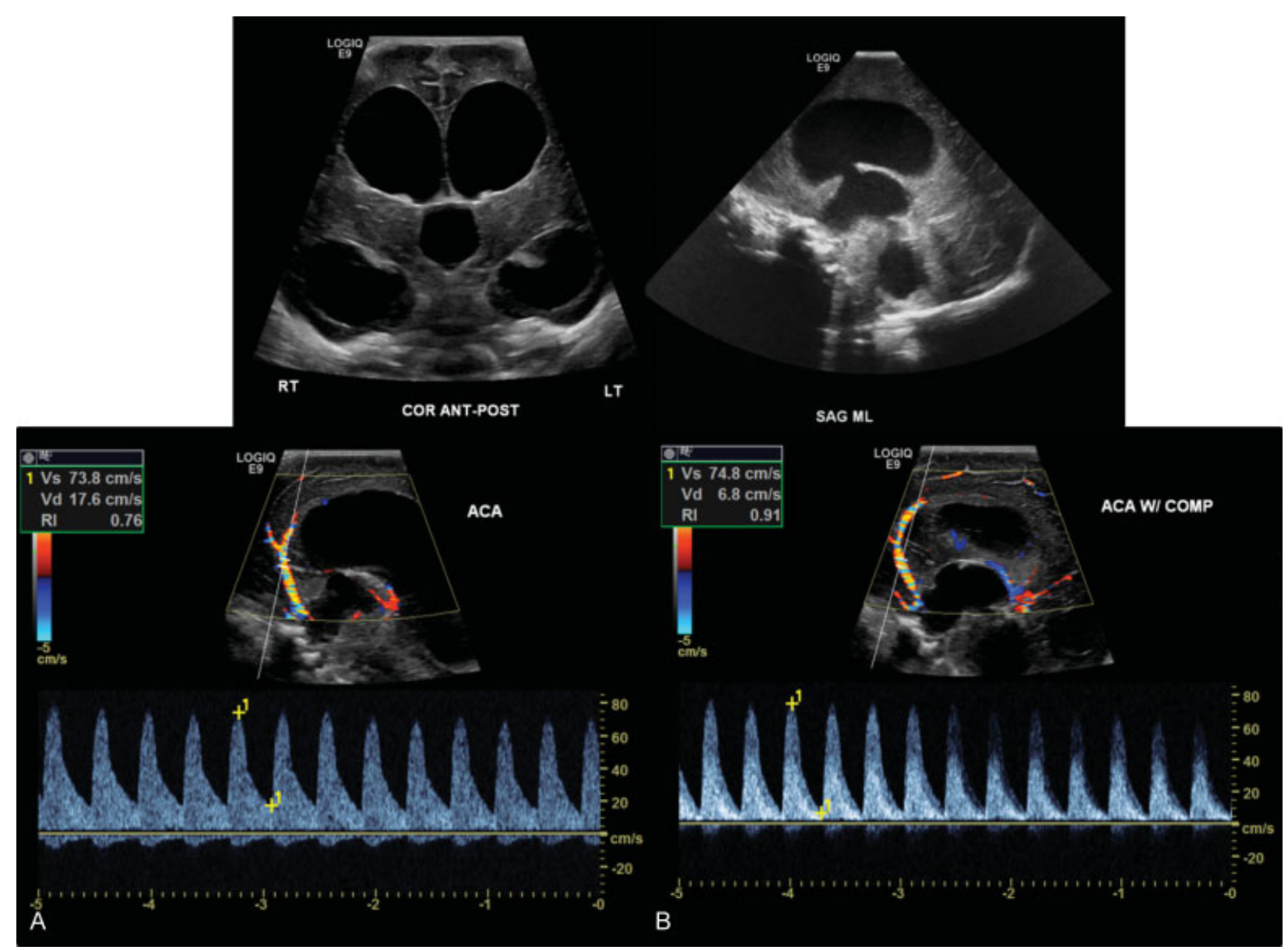

Fig. 22 Abnormal pulsed wave Doppler of ACA in the setting of preterm infant with posthemorrhagic hydrocephalus. (A) Normal ACA RI in preterm infant $(<0.85)$ at rest, $(B)$ increased ACA RI with compression of anterior fontanel which persists beyond 10 seconds, indicating loss of normal cerebral autoregulation in the setting of increased ICP, and (C) pulsed wave Doppler of ACA in the same infant following intraventricular shunt placement demonstrates normal ACA RI with compression of anterior fontanel, indicating relief of elevated ICP. ACA, anterior cerebral artery; ICP, intracranial pressures; RI, resistive index. 


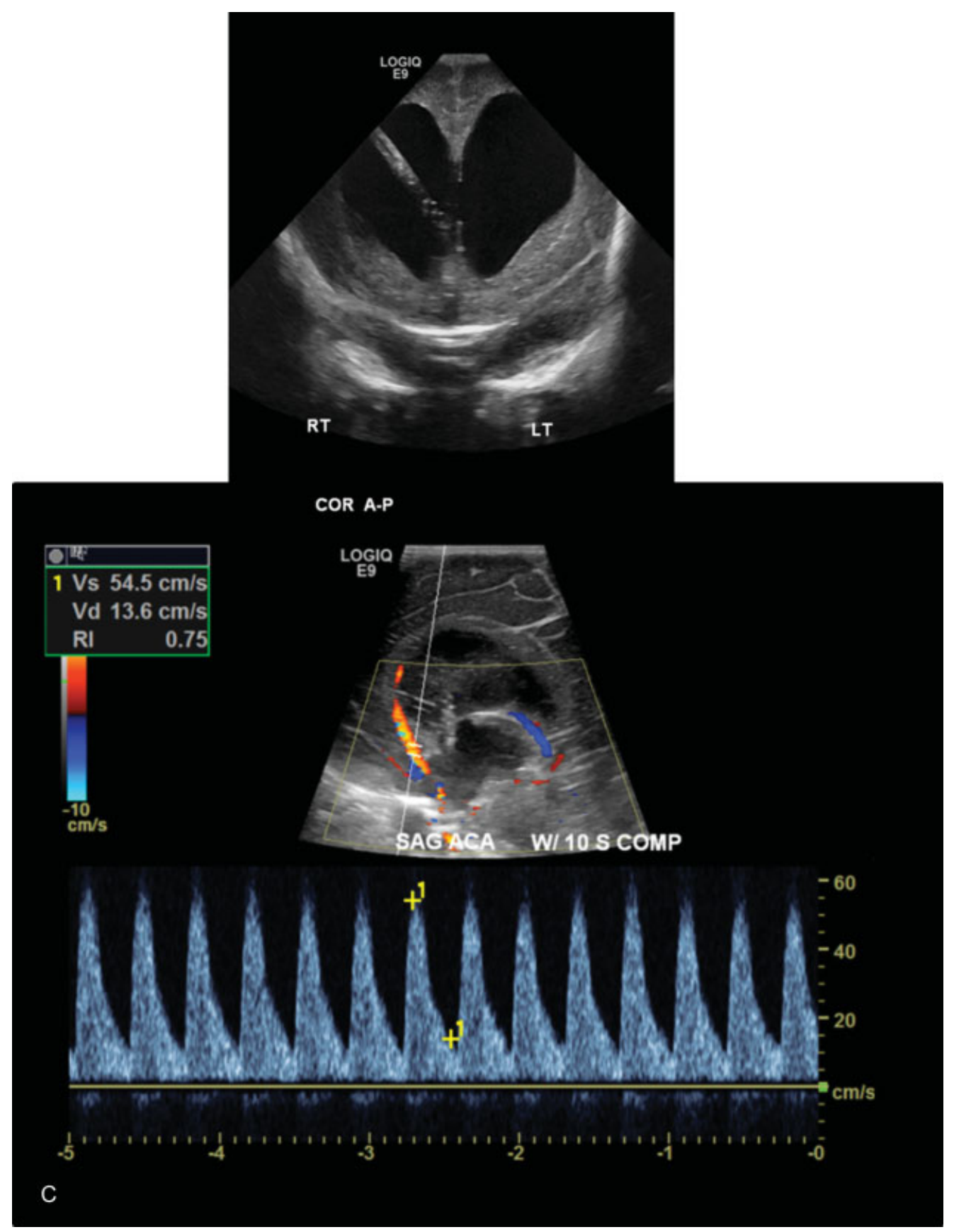

Fig. 22 (Continued)

change the cerebral blood flow velocities. Alteration of the RI by more than $45 \%$ between baseline and imaging with compression is suggestive of baseline elevated ICP and sustained elevation of the RI beyond 5 seconds can be used to predict which patients would benefit from ventricular shunt placement ( -Fig. 22A, B) ${ }^{7}$

The use of ACA Doppler evaluation in infants with ventriculomegaly is clinically helpful as change on Doppler often precedes clinical symptoms by days to weeks., ${ }^{2,7}$ Doppler evaluation may also be used to monitor therapeutic effect of intraventricular shunting and further guide clinical care (-Fig. 22C).

\section{Neonatal Meningitis}

Bacterial meningitis is a rare but serious condition in the newborn that occurs as a result of bacteremia and sepsis. The most common organisms associated with meningitis in the newborn are group B streptococcus, Escherichia coli, and Listeria monocytogenes. Infection spreads to the CNS via the highly vascular choroid plexus and in turn spreads to the CSF resulting in ventriculitis, meningitis, and arachnoiditis.

Early diagnosis and recognition of complications are imperative to prevention of neurodevelopmental sequelae. ${ }^{14}$ Cranial ultrasound is an excellent imaging modality 


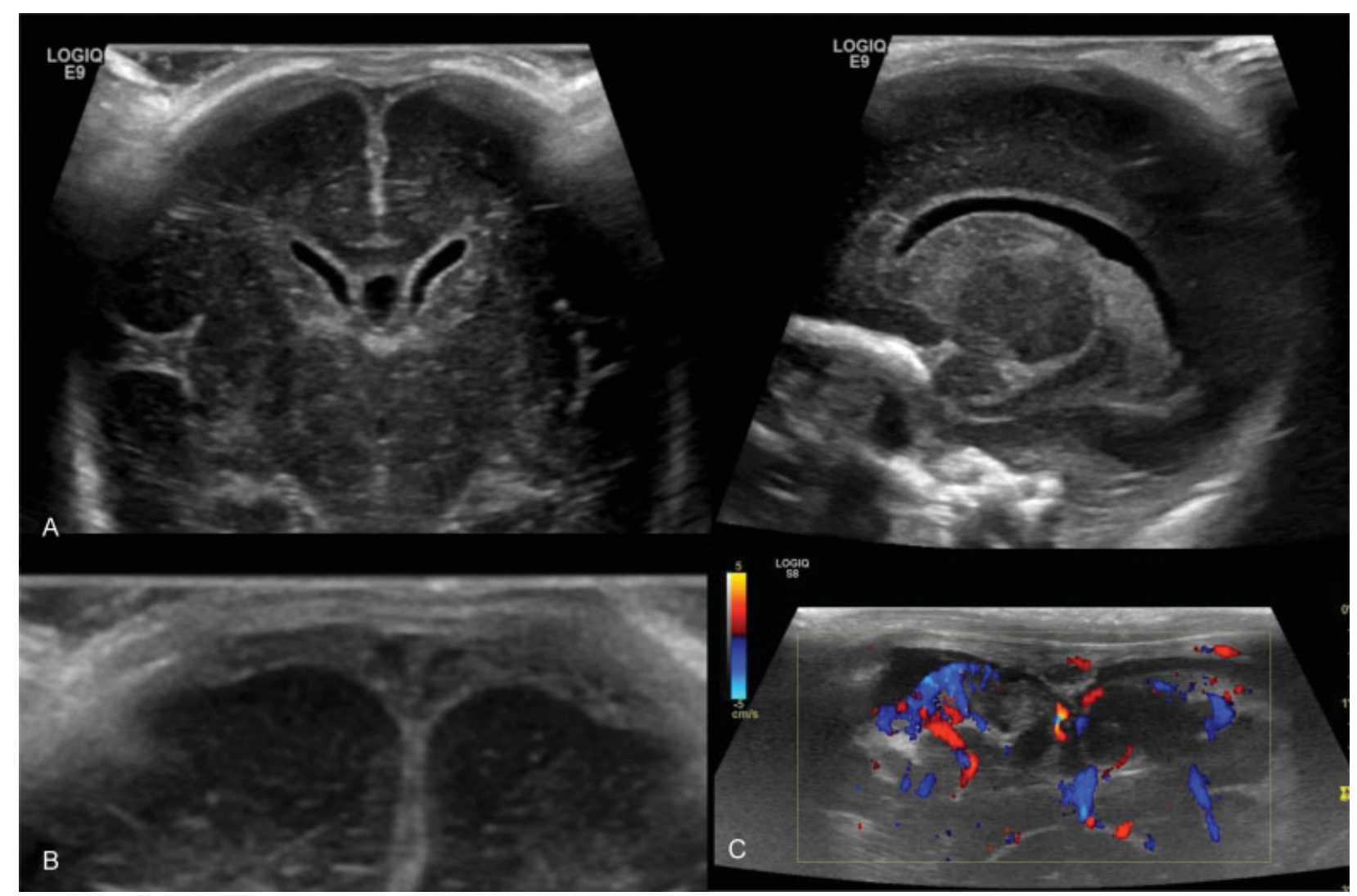

Fig. 23 Cranial ultrasound findings in neonatal meningitis. (A) Coronal and sagittal grayscale images with high frequency linear transducer demostrate thickening and increased echogenicity of the ependyma in both lateral ventricles consistent with ventriculitis. (B) Images optimized for evaluation of the extra-axial space demonstrates complex, echogenic fluid consistent with empyema in patient with Escherichia coli meningitis. (C) Color Doppler evaluation of the superior sagittal sinus in a different patient demonstrates echogenic intraluminal thrombus with lack of color flow.

for identification of important early findings including thickening and increased echogenicity of the meninges and ependymal lining of the ventricles cerebral edema ( - Fig. 23A). Ventriculomegaly can be seen in the acute or chronic phase of disease due to impaired CSF drainage. Color Doppler may demonstrate increased flow in the pial and subarachnoid vessels (- Fig. 23B). These findings are all seen to best effect with imaging using a high-frequency linear transducer.

A rare but significant complication of meningitis includes intracranial empyema with echogenic debris seen in the ventricles and extra-axial spaces (-Fig. 23C). Superior sagittal sinus venous thrombosis results from direct contact of subdural collections with the cortical vessels and venous sinuses, leading to thrombus and venous infarct. ${ }^{18}$

\section{Extra-axial Spaces}

Advanced cranial ultrasound techniques including imaging with high-frequency linear transducer and color Doppler has significantly improved ultrasound evaluation of the extraaxial spaces.

In normal, term infants, the width of the subarachnoid space ranges from 0 to $3 \mathrm{~mm}$ with a mean width of $1.6 \mathrm{~mm}$. $^{6}$
The extra-axial spaces may become enlarged in several clinical situations in the neonate including the setting of communicating hydrocephalus or underlying brain atrophy. Benign enlargement of the subarachnoid spaces in infancy may also be seen in older infants with macrocephaly. ${ }^{9}$ On grayscale ultrasound, simple anechoic fluid can be seen in the subarachnoid space deep to the anterior fontanel which is contiguous with fluid extending along the midline falx. Color Doppler imaging demonstrates normal vessels running along the cortical surface of the brain as well as small caliber vessels crossing the subarachnoid space (-Fig. 24).

Subdural hemorrhage can result from birth trauma. Accumulation of blood products in the subdural space will cause inferior displacement of the arachnoid membrane from the dura along the inner table of the calvarium. Grayscale ultrasound with high-frequency linear transducer can identify the displaced linear, hyperechoic arachnoid membrane (-Fig. 25). ${ }^{6}$ Acute subdural blood products will appear hyperechoic, while subacute or chronic blood products may appear hypo- to anechoic, which may make diagnosis difficult. The addition of color Doppler images can identify a lack of crossing vessels in the subdural space (-Fig. 25), confirming subdural fluid collection. 


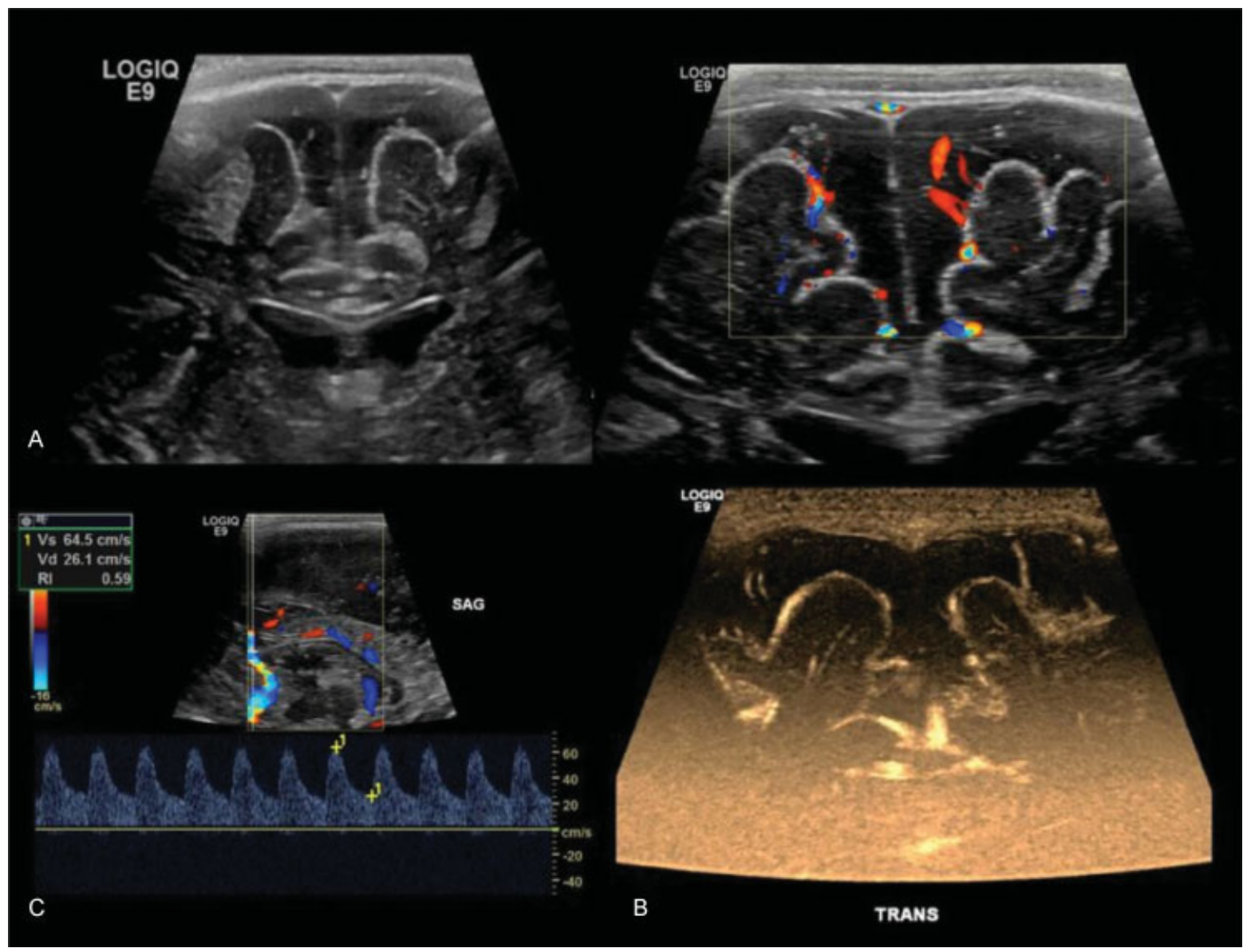

Fig. 24 Extra-axial space. Optimized images through the anterior fontanel using high-frequency linear transducer demonstrate classic findings of BESSI including (A) enlarged subarachnoid space with normal crossing arachnoid vessels, (B) B-flow imaging of the extra-axial space can better depict small crossing vessels, and (C) pulsed wave Doppler of the ACA demonstrates normal RI indicative of normal ICP. ACA, anterior cerebral artery; BESSI, benign enlargement of the subarachnoid spaces in infancy; ICP, intracranial pressures; RI, resistive index.

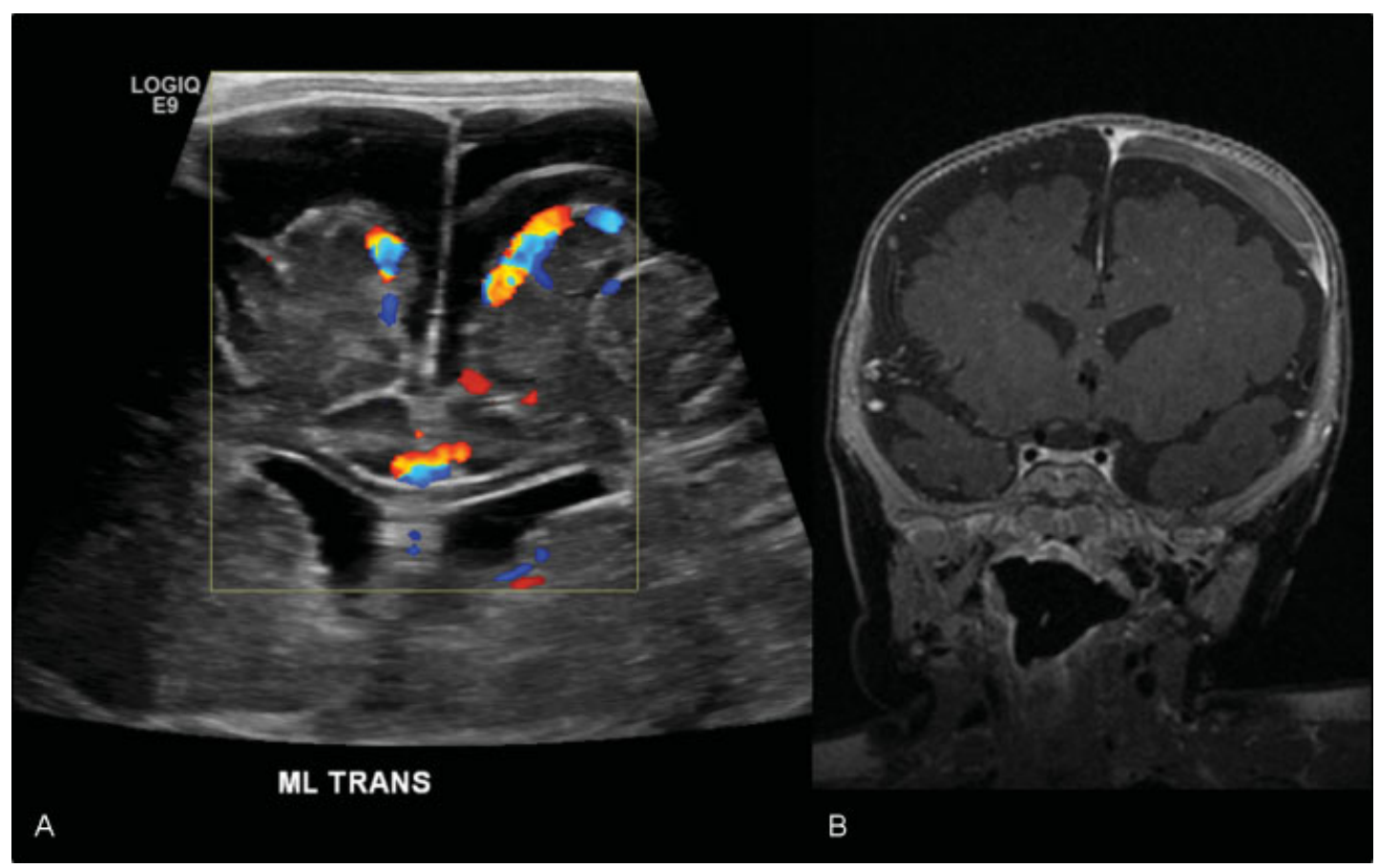

Fig. 25 Extra-axial space. Optimized images through the anterior fontanel using high-frequency linear transducer demonstrate findings of subacute subdural hemorrhage. (A) Inferior displacement of linear, hyperechoic arachnoid membranes in the right extra-axial space. Note crossing vessels in the deep subarachnoid space and lack of crossing vessels in the subdural space, (B) findings are confirmed on MRI. MRI, magnetic resonance imaging. 


\section{Conclusion}

Neonatal cranial ultrasound is an excellent tool for imaging of intracranial pathology in the preterm and term neonates. The addition of advanced techniques such as imaging with high-frequency linear transducers, additional acoustic windows, and Doppler can improve image quality and diagnostic accuracy of cranial ultrasound in the common pathologies such as GM/IVH, HII, cerebellar injury, ventriculomegaly, neonatal meningitis, and subdural hemorrhage.

\section{References}

1 Rosenberg HK, Angtuaco TL, Paltiel HG, Bulas DI, et al. ACR-AIUMSPR-SRU Practice Parameter for the Performance of Neurosonography in Neonates and Infants. Available at https://www.acr.org/ r/media/ACR/Documents/PGTS/guidelines/US_Neurosonography.pdf

2 Fritz J, Polansky SM, O'Connor SC. Neonatal neurosonography. Semin Ultrasound CT MR 2014;35(04):349-364

3 Daneman A, Epelman M. Neurosonography: in pursuit of an optimized examination. Pediatr Radiol 2015;45(Suppl 3):S406-S412

4 Lowe LH, Bailey Z. State-of-the-art cranial sonography: part 1, modern techniques and image interpretation. Am J Roentgenol 2011;196(05):1028-1033

5 Steggerda SJ, Leijser LM, Walther FJ, van Wezel-Meijler G. Neonatal cranial ultrasonography: how to optimize its performance. Early Hum Dev 2009;85(02):93-99

6 Di Salvo DN. A new view of the neonatal brain: clinical utility of supplemental neurologic US imaging windows. Radiographics 2001;21(04):943-955

7 Taylor GA, Madsen JR. Neonatal hydrocephalus: hemodynamic response to fontanelle compression- correlation with intracranial pressure and need for shunt placement. Radiology 1996;201(03): 685-689
8 Hintz SR, O'Shea M. Neuroimaging and neurodevelopmental outcomes in preterm infants. Semin Perinatol 2008;32(01):11-19

9 Seigel M. Pediatric Sonography. 3rd ed. Philadelphia, PA: Lippincott Williams and Wilkins; 2002

10 Daneman A, Epelman M, Blaser S, Jarrin JR. Imaging of the brain in full-term neonates: does sonography still play a role? Pediatr Radiol 2006;36(07):636-646

11 North K, Lowe L. Modern head ultrasound: normal anatomy, variants, and pitfalls that may simulate disease. Ultrasound Clin 2009;4:497-512

12 Yikilmaz A, Taylor GA. Sonographic findings in bacterial meningitis in neonates and young infants. Pediatr Radiol 2008;38(02):129-137

13 O'Shea TM, Counsell SJ, Bartels DB, Dammann O. Magnetic resonance and ultrasound brain imaging in preterm infants. Early Hum Dev 2005;81(03):263-271

14 Archer LN, Levene MI, Evans DH. Cerebral artery Doppler ultrasonography for prediction of outcome after perinatal asphyxia. Lancet 1986;2(8516):1116-1118

15 Maalouf EF, Duggan PJ, Counsell SJ, et al. Comparison of findings on cranial ultrasound and magnetic resonance imaging in preterm infants. Pediatrics 2001;107(04):719-727

16 Epelman M, Daneman A, Kellenberger CJ, et al. Neonatal encephalopathy: a prospective comparison of head US and MRI. Pediatr Radiol 2010;40(10):1640-1650

17 Dinan D, Daneman A, Guimaraes CV, Chauvin NA, Victoria T, Epelman M. Easily overlooked sonographic findings in the evaluation of neonatal encephalopathy: lessons learned from magnetic resonance imaging. Semin Ultrasound CT MR 2014;35(06): 627-651

18 de Vries LS, Eken P, Dubowitz LM. The spectrum of leukomalacia using cranial ultrasound. Behav Brain Res 1992;49(01):1-6

19 Veyrac C, Couture A, Saguintaah M, Baud C. Brain ultrasonography in the premature infant. Pediatr Radiol 2006;36(07): 626-635

20 Taylor G. Doppler of the neonatal and infant brain. In: Rumack CM, Wilson SR, Charboneau JW et al, eds. Diagnostic Ultrasound, 3rd ed. St. Louis: Elsevier Mosby; 2004:1703-1722 